\title{
General Theory of electronic configuration of atoms
}

\author{
Claude Ziad Bayeh \\ EEE GROUP-R\&D Department-LEBANON
}

\begin{abstract}
The "General Theory of electronic configuration of atoms" is an original study introduced by the author in chemistry in 2004. In this paper, the author developed a new method to write the electronic configuration for any atom, regardless of whether it actually exists or not in nature. This new method is based on Quantum theory and on three new and original formulae introduced and developed by the author. This method can be used to gather information about any atom's properties: its period, its group, its peripheral number of electrons and its theoretical electronic peripheral configuration. The main advantage of this method is that one can immediately knows the information about an atom, by a simple hand calculation without the need of software. Even if the atomic number is huge (as $Z=123453$ ). This method can be used in general chemistry courses and it is an extremely efficient method used for teaching and in the exam.

So any atomic number can be developed and we can find its electronic configuration regardless of whether it actually exists or not in nature.

-The traditional method of writing an electronic configuration is like this

$\widetilde{1 S^{2}} \overparen{2 S^{2} 2 P^{6}} \overparen{3 S^{2} 3 P^{6}} \overparen{4 S^{2} 3 D^{10} 3 P^{6}} \overbrace{5 S^{2} 4 D^{10} 5 P^{6}} \overbrace{6 S^{2} 4 F^{14} 5 D^{10} 6 P^{6}} \ldots$ Until finding the peripheral electronic configuration.

So the new method developed in this paper is mainly works on the peripheral electronic configuration without passing through the traditional method. It gives us directly the peripheral electronic configuration, for example

$\overbrace{6 S^{2} 4 F^{14} 5 D^{10} 6 P^{6}}$

In this way we have eliminated a very long process of calculation. This is a big advantage for the proposed method ahead the traditional one.

The main goal of introducing this paper is to reduce the calculation of obtaining the main information about an atom for example its period, group, number of electrons in the peripheral configuration and finding its peripheral electronic configuration as fast as possible even if the atom doesn't exist in reality. This paper doesn't explain the relativistic effects, because it is not the main goal of the proposed theory. We can still obtain the information about any atom without considering the relativistic effects.
\end{abstract}

Keywords: Electronic configuration, quantum theory, orbital, atom, electron.

\section{Introduction}

In the past, many scientists and researchers have developed several methods in order to calculate the electronic configuration of atoms [1,11,15, and 19]. These methods are very important and efficient, but the main problem is how to use a simple method of calculation without necessary software, and still get accurate results as fast as possible [20,21, 22, and 23]. This issue is now eliminated by a new method introduced and developed by the author. The concept of this method is based on three new formulae developed by the author in order to facilitate the calculation.

Moreover, this method gives birth to a new periodic table based on the theory, and the calculation which the name is "Bayeh's theoretical periodic table". This method can be used in general chemistry courses (especially in Quantum Chemistry) to calculate any electronic configuration even in some cases where it is impossible using the traditional methods.

In the second section, an overview on the general theory of electronic configuration is presented. Then in the third section, a theorem of ground state electron configuration is developed. In the fourth section, a calculation of the period of an element is introduced. In the section 5, some applicable examples are given in order to understand the theory. In sections 6 and 7, the theory is programmed in C++ language and in Visual Basic.NET with some examples. In the section 8 , a formulation of the theoretical periodic table is developed. Finally the conclusion is presented in the section 9 .

\section{Overview On The General Theory Of Electronic Configuration Of Atoms}

In this section, a simple example is introduced with an explanation in order to allow the reader to easily understand the upcoming theoretical part. The theory that was initially introduced by the author was also adapted by many advanced professors in the field; moreover they used it as a very reliable tool to help the 
students understand exactly how the configuration of electrons work and how to calculate the number of electrons, the period and the electronic configuration without using any software.

\section{Example with Atomic number equal to $\mathrm{Z}=\mathbf{2 0 3}$}

There exist only four steps that determine the period of the atom, the number of its peripheral electrons, the group of the atom and the ground state electron configuration:

\section{Step 1:}

By applying the equation (6) in the section 4.1 , we have to verify the equality

$\sum_{i=2}^{\sqrt{N}} i^{2}(P)==\frac{Z-2}{2}=\frac{203-2}{2}=100.5$

Where $P=1$ or $P=2$.

$i$ Is a variable that takes values from 2 to $\sqrt{N}$, and it is an image of the orbital that contains electrons.

$N$ Represents the last orbital containing peripheral electrons.

\section{Step 2:}

This step helps us determine which equation to apply amongst the equations 13,14, 15 or 16 presented in the section 4.2.

-Firstly, one has to calculate the sum $2 * 2^{2}+2 * 3^{2}+2 * 4^{2} \ldots(P) * \sqrt{N}^{2}$ that must be less or equal to

$\sum_{i=2}^{\sqrt{N}} i^{2}(P)=100.5$

E.g.: $\left(2 * 2^{2}+2 * 3^{2}+2 * 4^{2}+1 * 5^{2}\right)=83<\frac{z-2}{2}=100.5$

-then, calculate the sum $2 * 2^{2}+2 * 3^{2}+2 * 4^{2} \ldots(P) * \sqrt{N}^{2}$ that must be greater or equal to $\sum_{i=2}^{\sqrt{N}} i^{2}(P)=$ 100.5

E.g.: $\left(2 * 2^{2}+2 * 3^{2}+2 * 4^{2}+2 * 5^{2}\right)=108>\frac{Z-2}{2}=100.5$

These two equations above can be written in one equation as the following

$\sum_{i=2}^{\sqrt{N}} i^{2}(P)==\left\{\begin{array}{c}2 * 2^{2}+2 * 3^{2}+2 * 4^{2}+1 * 5^{2}=83<\frac{Z-2}{2}=100.5 \\ 2 * 2^{2}+2 * 3^{2}+2 * 4^{2}+(P=2) * 5^{2}=108>\frac{Z-2}{2}=100.5\end{array}\right.$

In this case we can deduce that $P$ is equal to 2 because it is the last number that is multiplied by 5 which is the image of the last orbital.

Once the value of $P$ is found, the period of the element is easily deduced, by the following method (that will be explained later);

Setting $v=P+1=3$ and $n=2 i-v ; P ;(2) /(1)$

$\Rightarrow n=2 * 5-1=9$ Which is the period of the atom, therefore the period is odd.

Step 3:

In this step, the number of peripheral electrons is obtained, and this number also determines the number of electrons remaining in the last orbital.

Calculation of the number of peripheral electrons will be as the following;

$N_{p e}=Z-\left(2+2 \sum_{i=2}^{\sqrt{N}} i^{2}(P)\right)=203-\left(2+2\left(\sum_{i=2}^{5} i^{2}(P)\right)\right.$

$$
=203-\left(2+2\left(2 * 2^{2}+2 * 3^{2}+2 * 4^{2}+1 * 5^{2}\right)=35\right.
$$

Therefore the Number of peripheral electrons is equal to $N_{p e}=35$.

Step 4:

The final step is to write the electron configuration of the last line (ground state electron configuration). As the number of peripheral electron as well as the period is given, one can easily find the electron configuration. The following formulae will be developed in the following sections. 


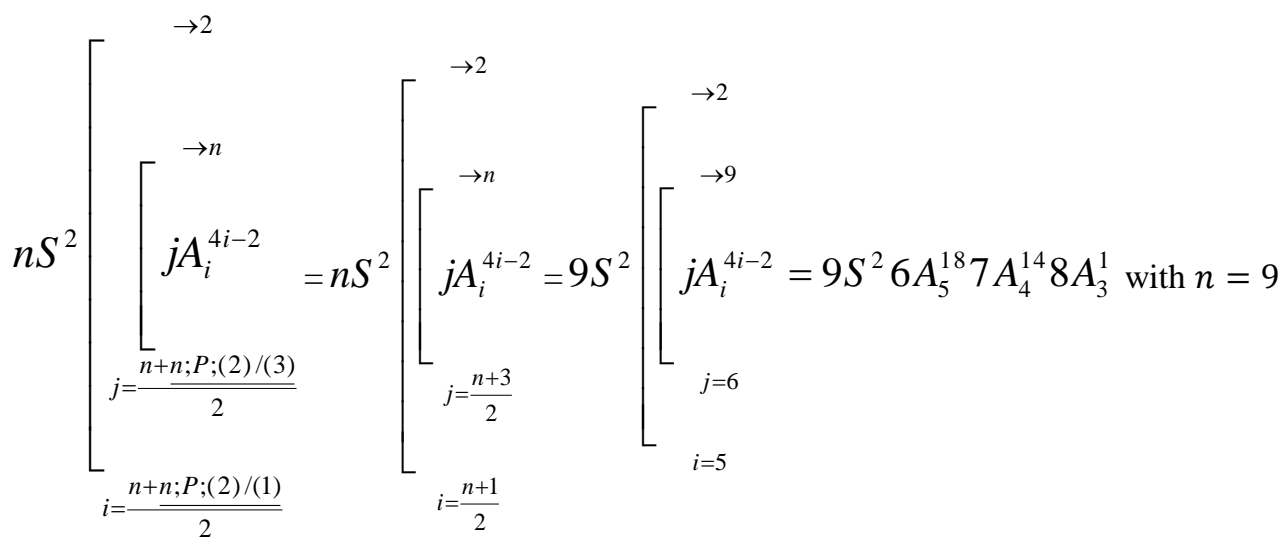

Briefly the Atom with $\mathrm{Z}=203$ has the following characteristic

- Period $n=9$

○ Number of peripheral electrons $N_{p e}=35$

$\circ \quad$ Group is $D^{9}=A_{3}^{1}$ (its location is presented on the picture below)

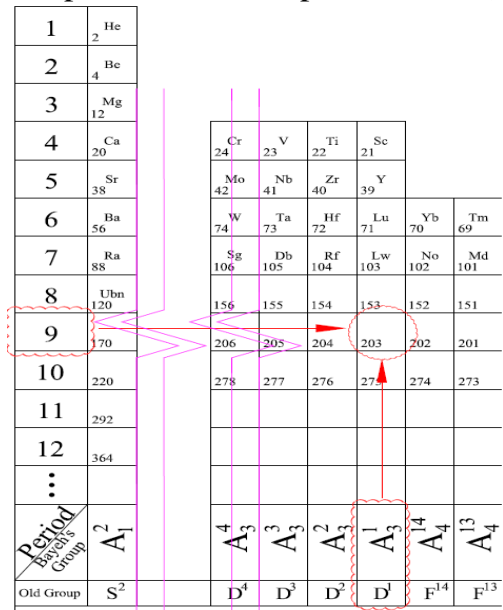

Fig. 1: The Atom with $\mathrm{Z}=203$ is presented in the circle, it is in the period 9 and group $D^{1}$ or $A_{3}^{1}$ of the Bayeh's theoretical periodic table of elements.

\section{Theorem Of The Ground State Electron Configuration}

Examples of the theorem will follow beginning in section 5.

Let's consider the following orbital: $\mathrm{S}^{2} \mathrm{P}^{6} \mathrm{D}^{10} \mathrm{~F}^{14} \mathrm{G}^{18} \mathrm{H}^{22} \mathrm{I}^{26} \mathrm{~J}^{30} \mathrm{~K}^{34} \mathrm{~L}^{38} \mathrm{M}^{42} \mathrm{~N}^{46} \ldots \ldots \ldots A_{n}^{2+4(n-1)}$

in which the inferior index $n$ of $A_{n}^{2+4(n-1)}$ indicates the period of the atom and the superior index $(2+4(n-1))$ indicates the maximum number of electron on the orbital $A_{n}^{2+4(n-1)}$.

$1 \mathrm{~S}^{2}$

$2 \mathrm{~S}^{2} \mathrm{P}^{6}$

$3 \mathrm{~S}^{2} \mathrm{P}^{6} \mathrm{D}^{10}$

$4 \mathrm{~S}^{2} \mathrm{P}^{6} \mathrm{D}^{10} \mathrm{~F}^{14}$

$5 \mathrm{~S}^{2} \mathrm{P}^{6} \mathrm{D}^{10} \mathrm{~F}^{14} \mathrm{G}^{18}$

$6 \mathrm{~S}^{2} \mathrm{P}^{6} \mathrm{D}^{10} \mathrm{~F}^{14} \mathrm{G}^{18} \mathrm{H}^{22}$

$7 \mathrm{~S}^{2} \mathrm{P}^{6} \mathrm{D}^{10} \mathrm{~F}^{14} \mathrm{G}^{18} \mathrm{H}^{22} \mathrm{I}^{26}$

$8 \mathrm{~S}^{2} \mathrm{P}^{6} \mathrm{D}^{10} \mathrm{~F}^{14} \mathrm{G}^{18} \mathrm{H}^{22} \mathrm{I}^{26} \mathrm{~J}^{30}$

$9 \mathrm{~S}^{2} \mathrm{P}^{6} \mathrm{D}^{10} \mathrm{~F}^{14} \mathrm{G}^{18} \mathrm{H}^{22} \mathrm{I}^{26} \mathrm{~J}^{30} \mathrm{~K}^{34}$

$10 \mathrm{~S}^{2} \mathrm{P}^{6} \mathrm{D}^{10} \mathrm{~F}^{14} \mathrm{G}^{18} \mathrm{H}^{22} \mathrm{I}^{26} \mathrm{~J}^{30} \mathrm{~K}^{34} \mathrm{~L}^{38}$

$11 \mathrm{~S}^{2} \mathrm{P}^{6} \mathrm{D}^{10} \mathrm{~F}^{14} \mathrm{G}^{18} \mathrm{H}^{22} \mathrm{I}^{26} \mathrm{~J}^{30} \mathrm{~K}^{34} \mathrm{~L}^{38} \mathrm{M}^{42}$

$12 \mathrm{~S}^{2} \mathrm{P}^{6} \mathrm{D}^{10} \mathrm{~F}^{14} \mathrm{G}^{18} \mathrm{H}^{22} \mathrm{I}^{26} \mathrm{~J}^{30} \mathrm{~K}^{34} \mathrm{~L}^{38} \mathrm{M}^{42} \mathrm{~N}^{46}$ 
$\mathrm{n} \mathrm{S}^{2}$ $A_{n}^{2+4(n-1)}$

The general orbital formulae is $A_{n}^{2+4(n-1)}$

In fact, for

$\mathrm{n}=1, A_{n}^{2+4(n-1)}=\mathrm{S}^{2}$

$\mathrm{n}=2, A_{n}^{2+4(n-1)}=\mathrm{P}^{6}$

$\mathrm{n}=3, A_{n}^{2+4(n-1)}=\mathrm{D}^{10}$ and so on.

-The complete ground state electron configuration is:

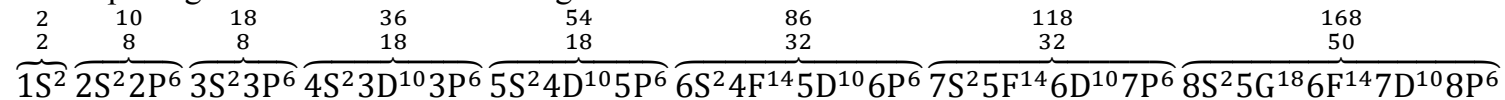

$\overbrace{9 \mathrm{~S}^{2} 6 \mathrm{G}^{18} 7 \mathrm{~F}^{14} 8 \mathrm{D}^{10} 9 \mathrm{P}^{6}}^{218} \overbrace{10 \mathrm{~S}^{2} 6 \mathrm{H}^{22} 7 \mathrm{G}^{18} 8 \mathrm{~F}^{14} 9 \mathrm{D}^{10} 10 \mathrm{P}^{6}}^{290} \overbrace{11 \mathrm{~S}^{2} 7 \mathrm{H}^{22} 8 \mathrm{G}^{18} 9 \mathrm{~F}^{14} 10 \mathrm{D}^{10} 11 \mathrm{P}^{6}}^{72}$

$\overbrace{12 \mathrm{~S}^{2} 7 \mathrm{I}^{26} 8 \mathrm{H}^{22} 9 \mathrm{G}^{18} 10 \mathrm{~F}^{14} 11 \mathrm{D}^{10} 12 \mathrm{P}^{6}}^{960} \overbrace{13 \mathrm{~S}^{2} 8 \mathrm{I}^{269 \mathrm{H}^{22}} 10 \mathrm{G}^{18} 11 \mathrm{~F}^{14} 12 \mathrm{D}^{10} 13 \mathrm{P}^{6}}^{98} \ldots \overbrace{\mathrm{nS}^{2} \ldots \ldots \ldots \ldots \mathrm{nP}^{6}}^{\begin{array}{c}558 \\ \text { Sum of electrons of the precedent brackets } \\ \text { maximum of electron on each bracket }\end{array}}$

Remark: Bracket in English has the same meaning of Line in French

-In order to shorten and simplify the demonstration, and unify all these shells and electron configurations, the author have introduced and developed new and original formulae that will be presented in the following paragraphs; these formulae are used in order to write all electron configurations in one formula.

The following formulae are deduced from the complete electronic configuration (ground state electron configuration) with a complete number of electrons:

1- If the period is an even number then

$n S^{2}\left[\sum_{j=\frac{n+2}{2}}^{\rightarrow n} j A_{i=\frac{n+2}{2}}^{4 i-2} ; i=\frac{n+2}{2} \rightarrow 2\right.$ and $j=\frac{n+2}{2} \rightarrow n$; with $n \geq 2$ and $n$ is an even number, $n \in N^{*}$

where

$i$ is going from $\frac{n+2}{2}$ to 2 .

$j$ is going from $\frac{n+2}{2}$ to $n$.

The parameters $i$ and $j$ vary sinultaneously. The parameter $i$ is decreasing by 1 until it is equal to 2 , and $j$ is increasing by 1 until it is equal to $n$.

For a complete ground state electron configuration, this formula will become as the following (all electrons are presented): 


$$
n S^{2}\left[\sum_{j=\frac{n+2}{2}}^{\rightarrow n} j A_{i}^{4 i-2}=n S^{2}\left(\frac{n+2}{2}\right) A_{\frac{n+2}{2}}^{4 \frac{n+2}{2}-2}\left(\frac{n+2}{2}+1\right) A_{i-1}^{4\left(\frac{n+2}{2}-1\right)-2}\left(\frac{n+2}{2}+2\right) A_{i-2}^{4\left(\frac{n+2}{2}-2\right)-2}\left(\frac{n+2}{2}+3\right) A_{i-1}^{4\left(\frac{n+2}{2}-3\right)-2} \ldots . . n P^{6}\right.
$$

Examples:

$$
\begin{aligned}
& \text { For } n=6 \Rightarrow n S^{2}\left[\sum_{j=\frac{n+2}{2}}^{\rightarrow n} j A_{i}^{4 i-2}=6 S^{2}\left[\left[\begin{array}{l}
\rightarrow 2 \\
j A_{i}^{4 i-2}=6 S^{2} 4 F^{14} 5 D^{10} 6 P^{6}
\end{array}\right.\right.\right. \\
& i=\frac{n+2}{2} \\
& \text { For } n=8 \Rightarrow n S^{2}\left[\sum_{j=\frac{n+2}{2}}^{\rightarrow n} A_{i=\frac{n+2}{2}}^{4 i-2}=8 S^{2}[\underbrace{\rightarrow 8}_{j=5} j A_{i=5}^{4 i-2}=8 S^{2} 5 A_{5}^{18} 6 A_{4}^{14} 7 A_{3}^{10} 8 A_{2}^{6}\right.
\end{aligned}
$$

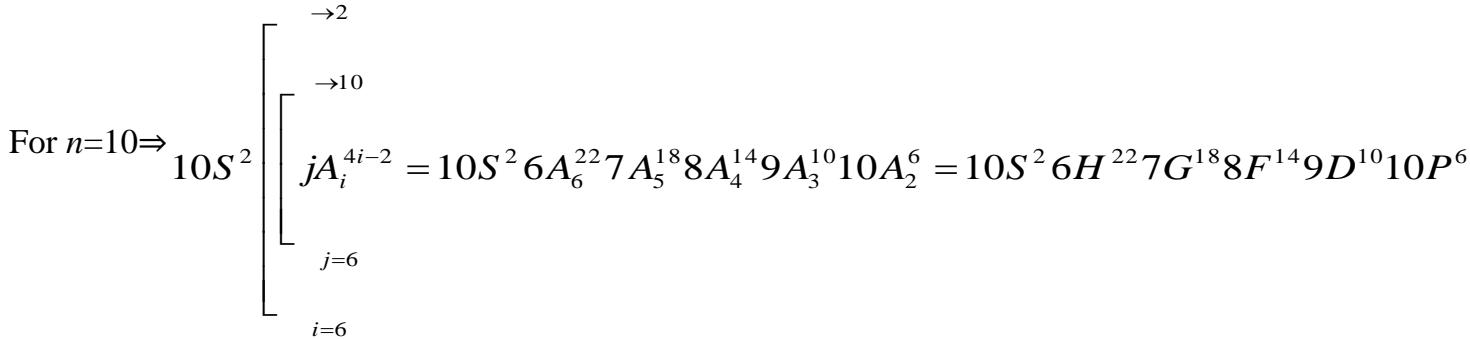

2- If the period is an odd number then

$$
n S^{2}[\underbrace{\rightarrow n}_{\substack{j=\frac{n+3}{2} \\ j A_{i}^{4 i-2}}} ; i=\frac{n+1}{2} \rightarrow 2 \text { and } j=\frac{n+3}{2} \rightarrow \mathrm{n} \text {; with } n \geq 1 \text { and } \mathrm{n} \text { is an odd number, } n \in N^{*}
$$


$i$ is going from $\frac{n+1}{2}$ to 2 .

$j$ is going from $\frac{n+3}{2}$ to $n$.

The parameters $i$ and $j$ vary simultaneously; The parameter $i$ is decreasing by 1 until it is equal to 2 , and $j$ is increasing by 1 until it is equal to $n$.

For a complete ground state electron configuration the form of this formula will be as the following (all electrons are presented):

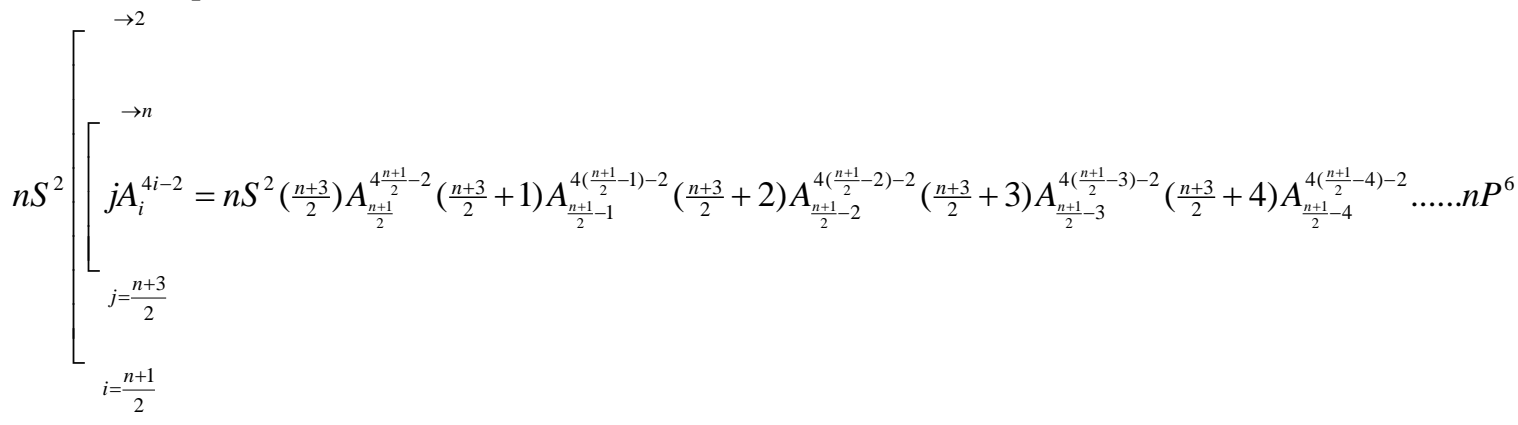

Examples:

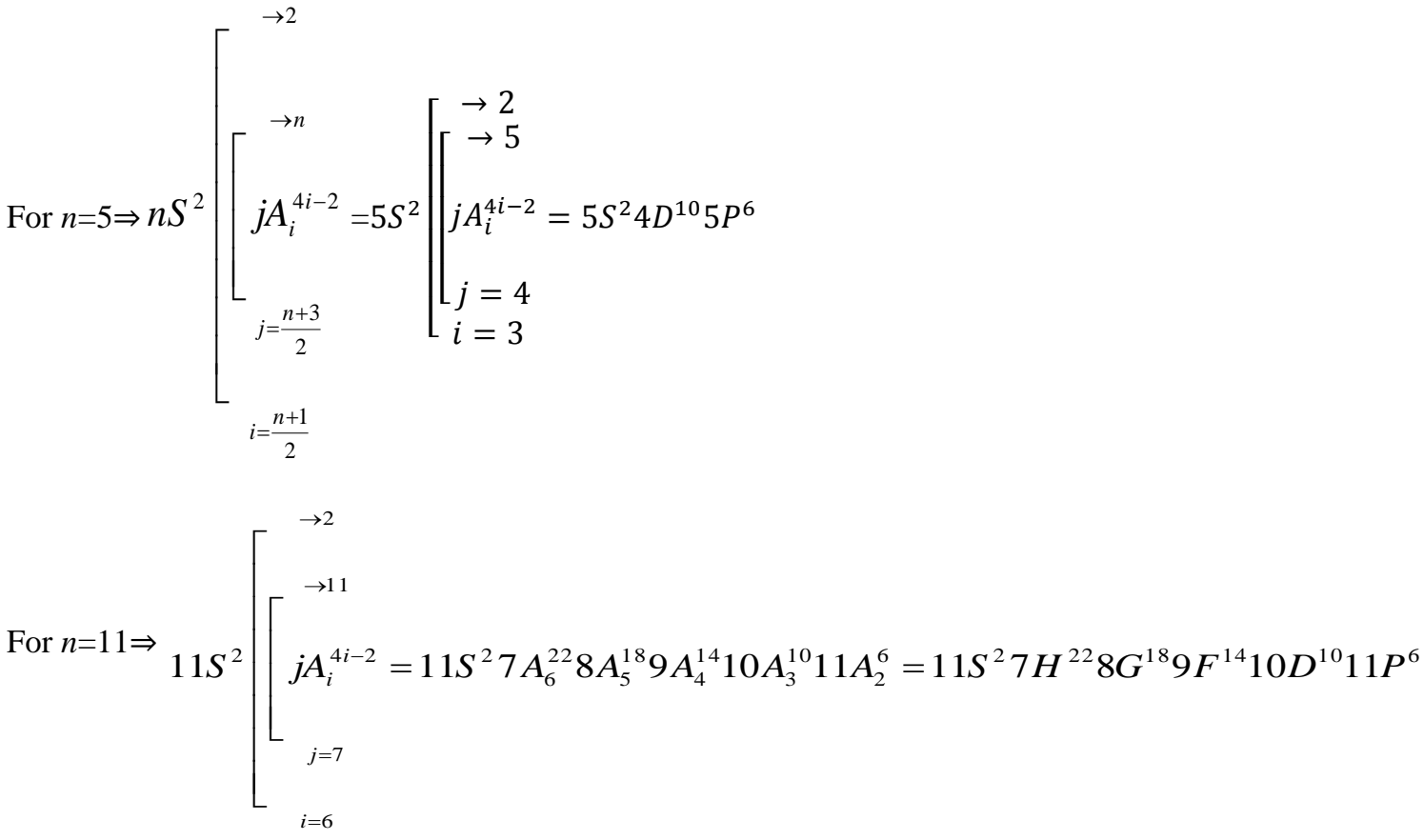

The two formulae presented above can be written in one formula using a function named "INFOMATH function" which also an original formula introduced by the author [26].

The general formula of the complete ground state electron configuration is written as the following:

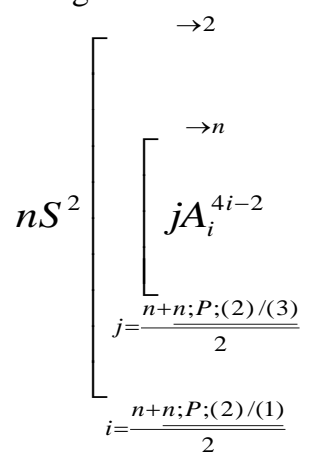


In the equation (3) there are two INFOMATH functions as following:

- $n ; P ;(2) /(1)$ is an INFOMATH function and can be read as the following:

If $n$ is an even number, then $\underline{n ; P ;(2) /(1)}=2$, else $\underline{n ; P ;(2) /(1)}=1$

Therefore, if $n$ is an even number, $i=\frac{n+2}{2}$, else $i=\frac{n+1}{2}$, just replace the value in the equation of $i=$ $\frac{n+n ; P ;(2) /(1)}{2}$.

- $n ; P ;(2) /(3)$ is an INFOMATH function and can be read as the following:

If $n$ is an even number, then $\underline{n ; P ;(2) /(3)}=2$, else $\underline{n ; P ;(2) /(3)}=3$

Therefore, if $n$ is even, $j=\frac{n+2}{2}$, else $j=\frac{n+3}{2}$ just replace the value in the equation of $j=\frac{n+n ; P ;(2) /(3)}{2}$.

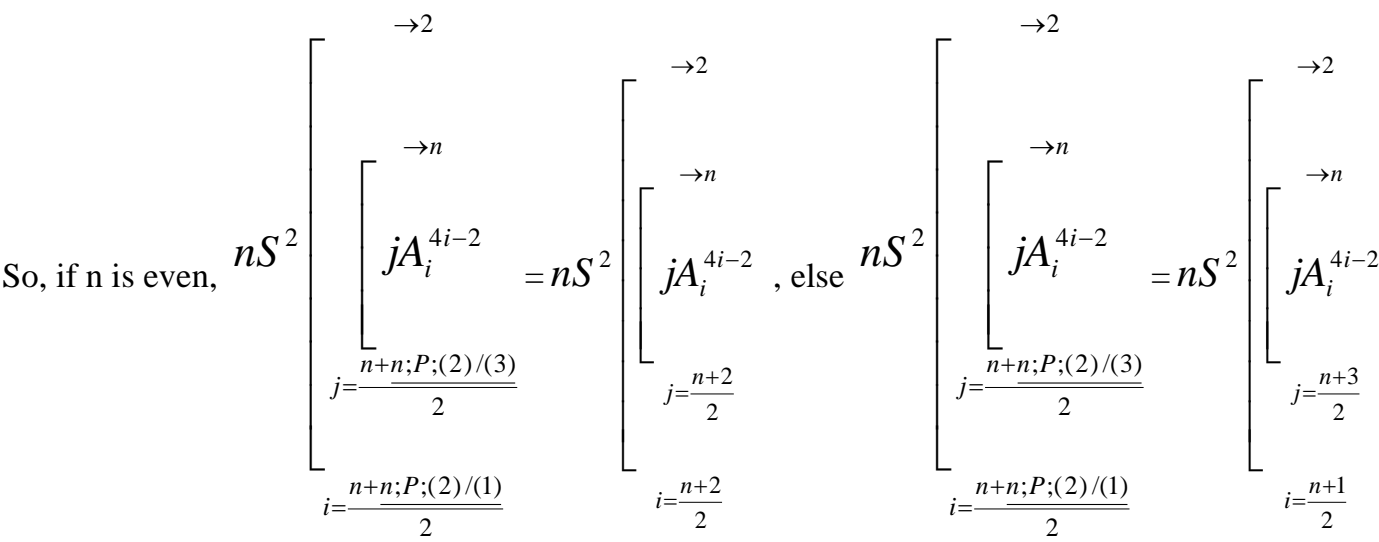

This verifies the equation (3)

\section{Calculattion Of The Period Of An Element}

In order to know the ground state configuration, one must know the period $(n)$ of the element. The following method allows knowing the period of the element, and therefore its ground state configuration.

The complete electronic configuration is:

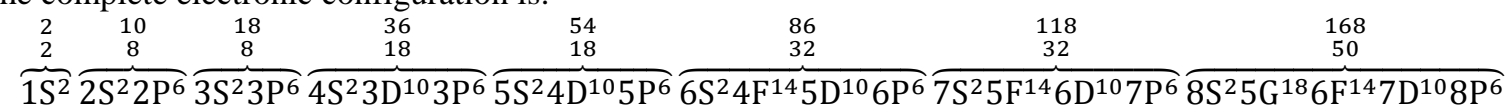

$\overbrace{9 \mathrm{~S}^{2} 6 \mathrm{G}^{18} 7 \mathrm{~F}^{14} 8 \mathrm{D}^{10} 9 \mathrm{P}^{6}}^{218} \overbrace{10 \mathrm{~S}^{2} 6 \mathrm{H}^{22} 7 \mathrm{G}^{18} 8 \mathrm{~F}^{14} 9 \mathrm{D}^{10} 10 \mathrm{P}^{6}}^{290} \overbrace{11 \mathrm{~S}^{2} 7 \mathrm{H}^{22} 8 \mathrm{G}^{18} 9 \mathrm{~F}^{14} 10 \mathrm{D}^{10} 11 \mathrm{P}^{6}}^{36}$

$\overbrace{12 \mathrm{~S}^{2} 7 \mathrm{I}^{26} 8 \mathrm{H}^{22} 9 \mathrm{G}^{18} 10 \mathrm{~F}^{14} 11 \mathrm{D}^{10} 12 \mathrm{P}^{6}}^{960} \overbrace{13 \mathrm{~S}^{2} 8 \mathrm{I}^{269 \mathrm{H}^{22}} 10 \mathrm{G}^{18} 11 \mathrm{~F}^{14} 12 \mathrm{D}^{10} 13 \mathrm{P}^{6}}^{98} \ldots \overbrace{\mathrm{nS}^{2} \ldots \ldots \ldots \ldots \mathrm{nP}^{6}}^{\begin{array}{c}958 \\ \text { sum of electrons of the precedent brackets } \\ \text { maximum of electron on each bracket }\end{array}}$

The maximal sum of the electrons on each line till the peripheral line is as the following:

$$
\begin{aligned}
S & =2+8+8+18+18+32+32+50+50+72+72+98+98+128+128+162+162 \ldots+\mathrm{N}+\mathrm{N}(\text { or just one } \mathrm{N}) \\
& =2+2 * 8+2 * 18+2 * 32+2 * 50+2 * 72+2 * 98+2 * 128+2 * 162+2 * 200+\ldots \ldots+(1 \text { or } 2) * \mathrm{~N} \\
& =2+2(8+18+32+50+72+98+128+162+200+\ldots .+(\mathrm{N}-1))+(1 \text { or } 2) * \mathrm{~N} \\
& =2+2\left(2 * 2^{2}+2 * 3{ }^{2}+2 * 4^{2}+2 * 5^{2}+2 * 6^{2}+2 * 7^{2}+2 * 8^{2}+2 * 9^{2}+\ldots+2 *\left(\sqrt{\frac{N-1}{2}}\right)^{2}\right)+(1 \text { or } 2) *(\sqrt{N})^{2} \\
& =2+2\left(2 * 2^{2}+2 * 3^{2}+2 * 4^{2}+2 * 5^{2}+2 * 6^{2}+\cdots+2 *\left(\sqrt{\frac{N-1}{2}}\right)^{2}\right)+(1 \text { or } 2) *(\sqrt{N})^{2} \\
& =2+2 \sum_{i=2}^{\sqrt{N}} i^{2}(1 \text { or } 2)
\end{aligned}
$$

Let's consider that $P=(1$ or 2$)$, then $S=2+2 \sum_{i=2}^{\sqrt{N}} i^{2}(P)$ 
Having $N$ as the number of electrons on the last line

-If the period $n$ is odd, then $n=2 i-1$

-If the period $n$ is even, then $n=2(i-1)$

These two formulae can be written in one formula by using the "INFOMATH function". Therefore, $n=2 i-v ; P ;(2) /(1)$, with $v=P+1$.

\section{IV.1. How to calculate $P$}

If the atomic number is given, equation (4) can be compared with the atomic number by the following equation:

$Z==2+2 \sum_{i=2}^{\sqrt{N}} i^{2}(P)$

The two equal signs (= =) indicate the comparison and verification of the number $\mathrm{Z}$ with the formula.

Therefore:

$\sum_{i=2}^{\sqrt{N}} i^{2}(P)==\frac{Z-2}{2}$

This formula gives two equations in which the atomic number will be situated in between the sum of each equation.

$\sum_{i=2}^{\sqrt{N}} i^{2}(P)=\left\{\begin{array}{l}2 * 2^{2}+2 * 3^{2}+2 * 4^{2}+2 * 5^{2}+\cdots+2 *\left(\sqrt{\frac{N-1}{2}}\right)^{2}+r *(\sqrt{N})^{2} \quad \text { (Inferior sum) } \\ 2 * 2^{2}+2 * 3^{2}+2 * 4^{2}+2 * 5^{2}+\cdots+2 *\left(\sqrt{\frac{N-1}{2}}\right)^{2}+(r+1) *(\sqrt{N})^{2} \text { (Superior sum) }\end{array}\right.$

Or it can be written in one equation:

$\sum_{i=2}^{\sqrt{N}} i^{2}(P)=2 * 2^{2}+2 * 3^{2}+2 * 4^{2}+2 * 5^{2}+\cdots+2 *\left(\sqrt{\frac{N-1}{2}}\right)^{2}+(P) *(\sqrt{N})^{2}$ (Superior sum)

With $r$ will take one of the two values $(r=0$ or $r=1)$ and $P=r+1$

For $r=0, P=1$ therefore:

$\sum_{i=2}^{\sqrt{N}} i^{2}(P)=2 * 2^{2}+2 * 3^{2}+\cdots+2 *\left(\sqrt{\frac{N-1}{2}}\right)^{2}+(1) *(\sqrt{N})^{2}$ (Superior sum)

For $r=1, P=2$ therefore:

$\sum_{i=2}^{\sqrt{N}} i^{2}(P)=2 * 2^{2}+2 * 3^{2}+\cdots+2 *\left(\sqrt{\frac{N-1}{2}}\right)^{2}+(2) *(\sqrt{N})^{2}$ (Superior sum)

In order to find $P$, one must look at the Superior sum and not at the Inferior sum. For this reason the following method contains 4 equations that verify the equality of the equation (6).

First equation:

$\sum_{i=2}^{\sqrt{N}} i^{2}(P)=\left\{\begin{array}{c}2 * 2^{2}+2 * 3^{2}+2 * 4^{2}+2 * 5^{2}+\cdots+2 *\left(\sqrt{\frac{N-1}{2}}\right)^{2} \quad \text { (Inferior sum) } \\ 2 * 2^{2}+2 * 3^{2}+2 * 4^{2}+2 * 5^{2}+\cdots+2 *\left(\sqrt{\frac{N-1}{2}}\right)^{2}+1 *(\sqrt{N})^{2} \text { (Superior sum) }\end{array}\right.$

With $P=1$, thus $v=P+1=2$ and $r=P-1=0$.

Second equation: 
$\sum_{i=2}^{\sqrt{N}} i^{2}(P)=\left\{\begin{array}{l}2 * 2^{2}+2 * 3^{2}+2 * 4^{2}+2 * 5^{2}+\cdots+2 *\left(\sqrt{\frac{N-1}{2}}\right)^{2}+1 *(\sqrt{N})^{2} \text { (Inferior sum) } \\ 2 * 2^{2}+2 * 3^{2}+2 * 4^{2}+2 * 5^{2}+\cdots+2 *\left(\sqrt{\frac{N-1}{2}}\right)^{2}+2 *(\sqrt{N})^{2} \text { (Superior sum) }\end{array}\right.$

With $P=2$, thus $v=P+1=3$ and $r=P-1=1$.

Third equation:

$\sum_{i=2}^{\sqrt{N}} i^{2}(P)=\left\{2 * 2^{2}+2 * 3^{2}+2 * 4^{2}+2 * 5^{2}+\cdots+2 *\left(\sqrt{\frac{N-1}{2}}\right)^{2}+1 *(\sqrt{N})^{2}\right.$ (Superior sum)

With $P=1$, thus $v=P+1=2$ and $r=P-1=0$.

Fourth equation:

$\sum_{i=2}^{\sqrt{N}} i^{2}(P)=\left\{2 * 2^{2}+2 * 3^{2}+2 * 4^{2}+2 * 5^{2}+\cdots+2 *\left(\sqrt{\frac{N-1}{2}}\right)^{2}+2 *(\sqrt{N})^{2}\right.$ (Superior sum)

With $P=2$, thus $v=P+1=3$ and $r=P-1=1$.

Briefly, To find the value of " $P$ ", always look at the (Superior sum), the following cases will appear:

-If $2 * 2^{2}+2 * 3^{2}+2 * 4^{2}+\cdots+2 *\left(\sqrt{\frac{N-1}{2}}\right)^{2}+1 *(\sqrt{N})^{2} \geq \frac{Z-2}{2}$ therefore, $P=1$

-If the $2 * 2^{2}+2 * 3^{2}+2 * 4^{2}+\cdots+2 *\left(\sqrt{\frac{N-1}{2}}\right)^{2}+2 *(\sqrt{N})^{2} \geq \frac{z-2}{2}$ therefore, $P=2$

\section{IV.2. Calculate $P$ from the Atomic number}

If the atomic number $(Z)$ is given, therefore the equation (5) and (6) are used,

In fact $\sum_{i=2}^{\sqrt{N}} i^{2}(P)==\frac{Z-2}{2}$ this equation leads to four different results as in equations $9,10,11$ and 12 . Therefore,

- First case:

$\sum_{i=2}^{\sqrt{N}} i^{2}(P)==\left\{\begin{array}{c}2 * 2^{2}+2 * 3^{2}+2 * 4^{2}+2 * 5^{2}+\cdots+2 *\left(\sqrt{\frac{N-1}{2}}\right)^{2}<\frac{Z-2}{2} \\ 2 * 2^{2}+2 * 3^{2}+2 * 4^{2}+2 * 5^{2}+\cdots+2 *\left(\sqrt{\frac{N-1}{2}}\right)^{2}+1 *(\sqrt{N})^{2}>\frac{Z-2}{2}\end{array}\right.$

With $P=1$, thus $v=P+1=2$ and $r=P-1=0$.

The value of $P$ can be find directly in the (Superior sum) of the equation (13) in which it takes the value that equals to 1 in $\left(1 *(\sqrt{N})^{2}\right)$.

In this case, $n=2 i-v ; P ;(2) /(1)$, with $v=P+1=2$.

$n=2 i-\underline{v ; P} ;(2) /(1)=2 i-2=2(i-1)$ this indicates that the period is even.

The same applies on the value of $i$ in which it takes the value of $\sqrt{N}$ (the last number in the equation (13), which represents the number of electrons in the last (Bracket) line).

$n=2 i-v ; P ;(2) /(1)=2 i-2=2(i-1)=2(\sqrt{N}-1)$

- Second case: 
$\sum_{i=2}^{\sqrt{N}} i^{2}(P)==\left\{\begin{array}{l}2 * 2^{2}+2 * 3^{2}+2 * 4^{2}+2 * 5^{2}+\cdots+1 *(\sqrt{N})^{2}<\frac{Z-2}{2} \\ 2 * 2^{2}+2 * 3^{2}+2 * 4^{2}+2 * 5^{2}+\cdots+2 *(\sqrt{N})^{2}>\frac{Z-2}{2}\end{array}\right.$

With $P=2$, thus $v=P+1=3$ and $r=P-1=1$.

The value of $P$ can be find directly in the (Superior sum) of the equation (14) in which it takes the value equal to 2 in $\left(2 *(\sqrt{N})^{2}\right)$.

In this case, $n=2 i-v ; P ;(2) /(1)$, with $v=P+1=3$.

$n=2 i-v ; P ;(2) /(1)=2 i-1$ this indicates that the period is odd.

The same applies on the value of $i$ which takes the value of $\sqrt{N}$ (the last number in the equation (14), which represents the number of electrons in the last (bracket) line).

$n=2 i-v ; P ;(2) /(1)=2 i-1=2 i-1=2 \sqrt{N}-1$

- Third case:

$\sum_{i=2}^{\sqrt{N}} i^{2}(P)==\left\{2 * 2^{2}+2 * 3^{2}+2 * 4^{2}+2 * 5^{2}+\cdots+2 *\left(\sqrt{\frac{N-1}{2}}\right)^{2}+1 *(\sqrt{N})^{2}=\frac{Z-2}{2}\right.$

With $P=1$, thus $v=P+1=2$ and $r=P-1=0$.

The value of $P$ can be found directly in the (Superior sum) of the equation (15) in which it takes the value equal to 1 in $\left(1 *(\sqrt{N})^{2}\right)$.

In this case, $n=2 i-v ; P ;(2) /(1)$, with $v=P+1=2$.

$n=2 i-\underline{v ; P} ;(2) /(1)=2 i-2=2(i-1)$ this indicates that the period is even.

The same applies on the value of $i$ which takes the value of $\sqrt{N}$ (the last number which represents the number of electrons in the last (bracket) line).

$n=2 i-\underline{v ; P}(2) /(1)=2 i-2=2(i-1)=2(\sqrt{N}-1)$

- Fourth case:

$\sum_{i=2}^{\sqrt{N}} i^{2}(P)==\left\{2 * 2^{2}+2 * 3^{2}+2 * 4^{2}+2 * 5^{2}+\cdots+2 *\left(\sqrt{\frac{N-1}{2}}\right)^{2}+2 *(\sqrt{N})^{2}=\frac{Z-2}{2}\right.$

With $P=2$, thus $v=P+1=3$ and $r=P-1=1$.

The value of $P$ can be found directly in the (Superior sum) of the equation (16) in which it takes the value equal to 2 in $\left(2 *(\sqrt{N})^{2}\right)$.

In this case, $n=2 i-v ; P ;(2) /(1)$, with $v=P+1=3$.

$n=2 i-\underline{v ; P ;(2) /(1)}=2 i-1$ this indicates that the period is odd.

The same applies on the value of $i$ which takes the value of $\sqrt{N}$ (the last number which represents the number of electrons in the last (bracket) line).

$n=2 i-v ; P ;(2) /(1)=2 i-1=2 i-1=2 \sqrt{N}-1$

Briefly, To find the value of $P$, always look at the (Superior sum), the following cases will appear:

-If $2 * 2^{2}+2 * 3^{2}+2 * 4^{2}+\cdots+2 *\left(\sqrt{\frac{N-1}{2}}\right)^{2}+1 *(\sqrt{N})^{2} \geq \frac{Z-2}{2}$

Therefore, $P=1 \Rightarrow$ Period is even

-If the $2 * 2^{2}+2 * 3^{2}+2 * 4^{2}+\cdots+2 *\left(\sqrt{\frac{N-1}{2}}\right)^{2}+2 *(\sqrt{N})^{2} \geq \frac{Z-2}{2}$

Therefore, $P=2 \Rightarrow$ Period is Odd 


\section{IV.3. Calculation of the peripheral number of electrons}

If the atomic number $(Z)$ is given, therefore the equation (5) is used; therefore the Number of peripheral electrons named $N_{p e}$ is equal to $N_{p e}=Z-\left(2+2 \sum_{i=2}^{\sqrt{N}} i^{2}(P)\right)$

In order to find the number $N$ and $P$ mentioned, we have to follow the equations $13,14,15$ and 16 which lead to the following cases:

- First case:

$\sum_{i=2}^{\sqrt{N}} i^{2}(P)==\left\{\begin{array}{c}2 * 2^{2}+2 * 3^{2}+2 * 4^{2}+2 * 5^{2}+\cdots+2 *\left(\sqrt{\frac{N-1}{2}}\right)^{2}<\frac{Z-2}{2} \\ 2 * 2^{2}+2 * 3^{2}+2 * 4^{2}+2 * 5^{2}+\cdots+2 *\left(\sqrt{\frac{N-1}{2}}\right)^{2}+1 *(\sqrt{N})^{2}>\frac{Z-2}{2}\end{array}\right.$

$\Rightarrow N_{p e}=Z-\left(2+2 \sum_{i=2}^{\sqrt{N}} i^{2}(P)\right)$

With $\sum_{i=2}^{\sqrt{N}} i^{2}(P)=2 * 2^{2}+2 * 3^{2}+2 * 4^{2}+2 * 5^{2}+\cdots+2 *\left(\sqrt{\frac{N-1}{2}}\right)^{2}$

Which is the lowest summation.

$\Rightarrow N_{p e}=Z-\left(2+2\left(2 * 2^{2}+2 * 3^{2}+2 * 4^{2}+2 * 5^{2}+\cdots+2 *\left(\sqrt{\frac{N-1}{2}}\right)^{2}\right)\right)$

- Second case:

$\sum_{i=2}^{\sqrt{N}} i^{2}(P)==\left\{\begin{array}{l}2 * 2^{2}+2 * 3^{2}+2 * 4^{2}+2 * 5^{2}+\cdots+1 *(\sqrt{N})^{2}<\frac{Z-2}{2} \\ 2 * 2^{2}+2 * 3^{2}+2 * 4^{2}+2 * 5^{2}+\cdots+2 *(\sqrt{N})^{2}>\frac{Z-2}{2}\end{array}\right.$

$\Rightarrow N_{p e}=Z-\left(2+2 \sum_{i=2}^{\sqrt{N}} i^{2}(P)\right)$

With $\sum_{i=2}^{\sqrt{N}} i^{2}(P)=2 * 2^{2}+2 * 3^{2}+2 * 4^{2}+2 * 5^{2}+\cdots+1 *(\sqrt{N})^{2}$

$\Rightarrow N_{p e}=Z-\left(2+2\left(2 * 2^{2}+2 * 3^{2}+2 * 4^{2}+2 * 5^{2}+\cdots+1 *(\sqrt{N})^{2}\right)\right)$

- Third case:

$\sum_{i=2}^{\sqrt{N}} i^{2}(P)==\left\{2 * 2^{2}+2 * 3^{2}+2 * 4^{2}+2 * 5^{2}+\cdots+2 *\left(\sqrt{\frac{N-1}{2}}\right)^{2}+1 *(\sqrt{N})^{2}=\frac{Z-2}{2}\right.$

$\Rightarrow N_{p e}=Z-\left(2+2 \sum_{i=2}^{\sqrt{N}} i^{2}(P)\right)$

With $\sum_{i=2}^{\sqrt{N}} i^{2}(P)=2 * 2^{2}+2 * 3^{2}+2 * 4^{2}+2 * 5^{2}+\cdots+2 *\left(\sqrt{\frac{N-1}{2}}\right)^{2}$ which is the internal line

$\Rightarrow N_{p e}=Z-\left(2+2\left(2 * 2^{2}+2 * 3^{2}+2 * 4^{2}+2 * 5^{2}+\cdots+2 *\left(\sqrt{\frac{N-1}{2}}\right)^{2}\right)\right)$

- Fourth case:

$\sum_{i=2}^{\sqrt{N}} i^{2}(P)==\left\{2 * 2^{2}+2 * 3^{2}+2 * 4^{2}+2 * 5^{2}+\cdots+2 *\left(\sqrt{\frac{N-1}{2}}\right)^{2}+2 *(\sqrt{N})^{2}=\frac{Z-2}{2}\right.$

$\Rightarrow N_{p e}=Z-\left(2+2 \sum_{i=2}^{\sqrt{N}} i^{2}(P)\right)$

With $\sum_{i=2}^{\sqrt{N}} i^{2}(P)=2 * 2^{2}+2 * 3^{2}+2 * 4^{2}+2 * 5^{2}+\cdots+2 *\left(\sqrt{\frac{N-1}{2}}\right)^{2}+1 *(\sqrt{N})^{2}$

$\Rightarrow N_{p e}=Z-\left(2+2\left(2 * 2^{2}+2 * 3^{2}+2 * 4^{2}+2 * 5^{2}+\cdots+2 *\left(\sqrt{\frac{N-1}{2}}\right)^{2}+1 *(\sqrt{N})^{2}\right)\right)($

Briefly, the Number of peripheral electrons $\left(N_{p e}\right)$ is equal to $N_{p e}=Z-\left(2+2 \sum_{i=2}^{\sqrt{N}} i^{2}(P)\right)$ where $\sum_{i=2}^{\sqrt{N}} i^{2}(P)$ is the internal sum of the inferior line. 


\section{IV.4. Construction of the ground state electron configuration}

When the period $n$ and the number of the peripheral electrons are known, one can construct the ground state electron configuration of the last peripheral line using equation (3).

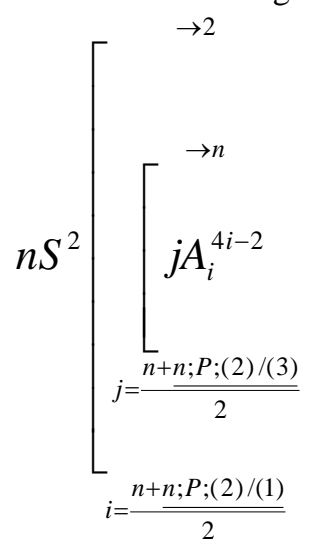

To fill the orbital with electron configuration you have to complete the expression in the above formula $\left(j A_{i}^{4 i-2}\right)$

Example 1: if the period is $n=4$, and you have 8 peripheral electrons, your final configuration will be as the following: $4 S^{2} 3 D^{6}$

Example 2: if the period is $n=8$, and you have 47 peripheral electrons, your final configuration will be as the following: $8 S^{2} 5 G^{18} 6 F^{14} 7 D^{10} 8 P^{3}$

\section{Application Examples Of The Theory}

This section is very important to understand the theory and its application in the chemistry domain. In this section, six examples are treated to cover all theories and cases.

Find the period, group, number of electron configuration and the electron configuration of the last line (ground state electron configuration) for the following atomic numbers: 47, 168, 203, 218, 365, and 10452.

\section{V.1. For $\mathrm{Z}=47$}

Step 1:

By equation (6), we have to verify the equality

$\sum_{i=2}^{\sqrt{N}} i^{2}(P)==\frac{Z-2}{2}=\frac{47-2}{2}=22.5$

Step 2:

We have to know exactly which the case applies to the atom amongst one of the equations $13,14,15$ or 16.

$\sum_{i=2}^{\sqrt{N}} i^{2}(P)=\left\{\begin{array}{l}2 * 2^{2}+1 * 3^{2}=17<\frac{Z-2}{2}=22.5 \\ 2 * 2^{2}+2 * 3^{2}=26>\frac{Z-2}{2}=22.5\end{array}\right.$

In this case we can deduce that $P$ is equal to 2 therefore $v=P+1=3$ and $n=2 i-v ; P ;(2) /(1)$ $\Rightarrow n=2 * 3-1=5$, which is the period of the atom; therefore the period is odd.

Step 3:

Calculation of the number of peripheral electrons:

$N_{p e}=Z-\left(2+2 \sum_{i=2}^{\sqrt{N}} i^{2}(P)\right)=47-\left(2+2\left(\sum_{i=2}^{\sqrt{N}} i^{2}(1)\right)=47-\left(2+2\left(2.2^{2}+1 \cdot 3^{2}\right)=11\right.\right.$

Therefore the number of peripheral electrons is equal to $N_{p e}=11$.

Step 4:

The final step is to write the electron configuration of the last line (ground state electron configuration). As the number of peripheral electron is given, the same for the period, one can find easily the electron configuration. 


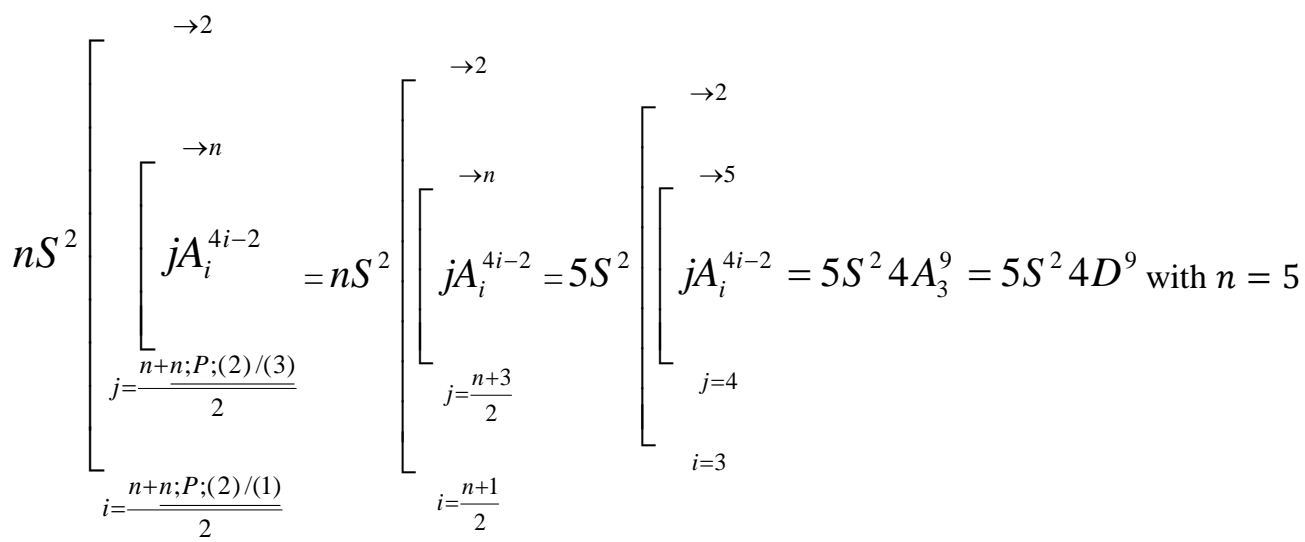

Briefly the Atom with $Z=47$ has the following characteristic

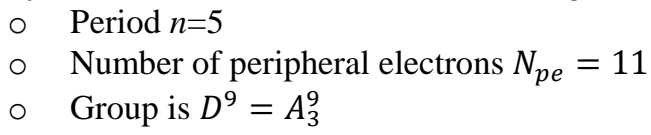

\section{V.2. For $Z=168$}

Step 1:

By equation (6), we have to verify the equality

$\sum_{i=2}^{\sqrt{N}} i^{2}(P)==\frac{Z-2}{2}=\frac{168-2}{2}=83$

Step 2:

We have to know exactly which the case applies to the atom, amongst one of the equations $13,14,15$ or 16.

$\sum_{i=2}^{\sqrt{N}} i^{2}(P)==\left\{2.2^{2}+2.3^{2}+2.4^{2}+1.5^{2}=83=\frac{Z-2}{2}=83\right.$

In this case we can deduce that $P$ is equal to 1 therefore $v=P+1=2$ and $n=2 i-v ; P ;(2) /(1) \Rightarrow n=2 *$ $5-2=8$, which is the period of the atom; therefore the period is even.

Step 3:

Calculation of the number of peripheral electrons will be as the following;

$$
\begin{aligned}
N_{p e} & =Z-\left(2+2 \sum_{i=2}^{4} i^{2}(P)\right)=168-\left(2+2\left(\sum_{i=2}^{4} i^{2}(2)\right)\right. \\
& =168-\left(2+2\left(2 * 2^{2}+2 * 3^{2}+2 * 4^{2}\right)=50\right.
\end{aligned}
$$

Therefore the number of peripheral electrons is equal to $N_{p e}=50$.

Step 4:

The final step is to write the electron configuration of the last line (ground state electron configuration) As the number of peripheral electron is given, the same for the period, one can find easily the electron configuration. 
$n S^{2}\left[\sum_{j=\frac{n+n ; P ;(2) /(3)}{2}}^{j A_{i}^{4 i-2}}=n S^{2}\left[\sum_{j=\frac{n+2}{2}}^{\rightarrow n} j A_{i=\frac{n+2}{2}}^{4 i-2}=8 S^{2}\left[\sum_{j=5}^{\rightarrow 2} j A_{i=5}^{4 i-2}=8 S^{2} 5 A_{5}^{18} 6 A_{4}^{14} 7 A_{3}^{10} 8 A_{2}^{6}\right.\right.\right.$ with $n=8$

Briefly the Atom with $Z=168$ has the following characteristic

- Period $n=8$

- Number of peripheral electrons $N_{p e}=50$

- Group is $A_{2}^{6}$ (it's location is presented on the picture below)

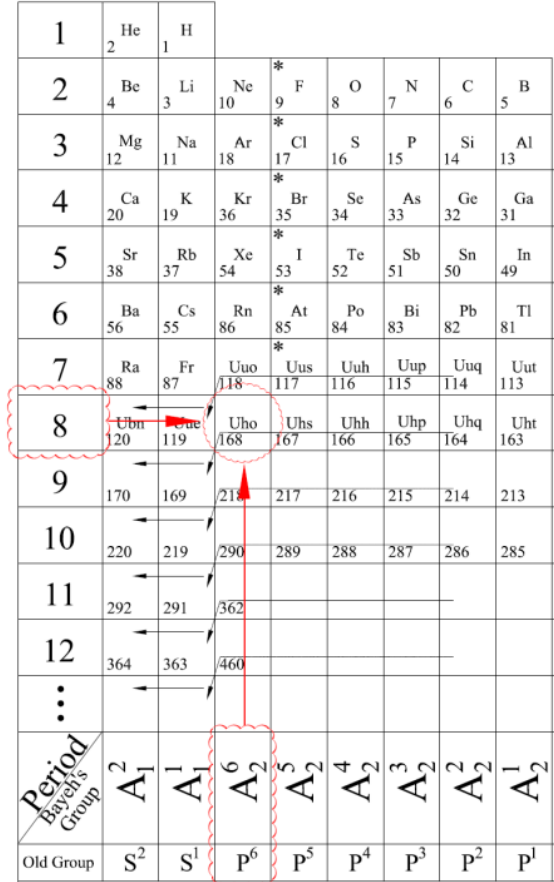

BAYEH's Theoretical Periodic table of

Fig. 2: The Atom with $\mathrm{Z}=168$ is presented in the circle, it is in the period 8 and group $P^{6}$ or $A_{2}^{6}$ of the Bayeh's theoretical periodic table of elements.

\section{V.3. For $Z=203$}

Step 1:

By equation (6), we have to verify the equality.

$\sum_{i=2}^{\sqrt{N}} i^{2}(P)==\frac{Z-2}{2}=\frac{203-2}{2}=100.5$

Step 2:

We have to know exactly which case applies to the atom amongst one of the equations 13,14,15 or 16 .

$\sum_{i=2}^{\sqrt{N}} i^{2}(P)==\left\{\begin{array}{l}2 * 2^{2}+2 * 3^{2}+2 * 4^{2}+1 * 5^{2}=83<\frac{Z-2}{2}=100.5 \\ 2 * 2^{2}+2 * 3^{2}+2 * 4^{2}+2 * 5^{2}=108>\frac{Z-2}{2}=100.5\end{array}\right.$

In this case we can deduce that $P$ is equal to 2 therefore $v=P+1=3$ and $n=2 i-v ; P ;(2) /(1) \Rightarrow n=2$ * $5-1=9$ which is the period of the atom; therefore the period is odd. 
Step 3:

Calculation of the number of peripheral electrons will be as the following:

$N_{p e}=Z-\left(2+2 \sum_{i=2}^{\sqrt{N}} i^{2}(P)\right)=203-\left(2+2\left(\sum_{i=2}^{5} i^{2}(P)\right)\right.$

$=203-\left(2+2\left(2 * 2^{2}+2 * 3^{2}+2 * 4^{2}+1 * 5^{2}\right)=35\right.$

Therefore the number of peripheral electrons is equal to $N_{p e}=35$.

Step 4:

The final step is to write the electron configuration of the last line (ground state electron configuration) As the number of peripheral electron is given, the same for the period, one can find easily the electron configuration.

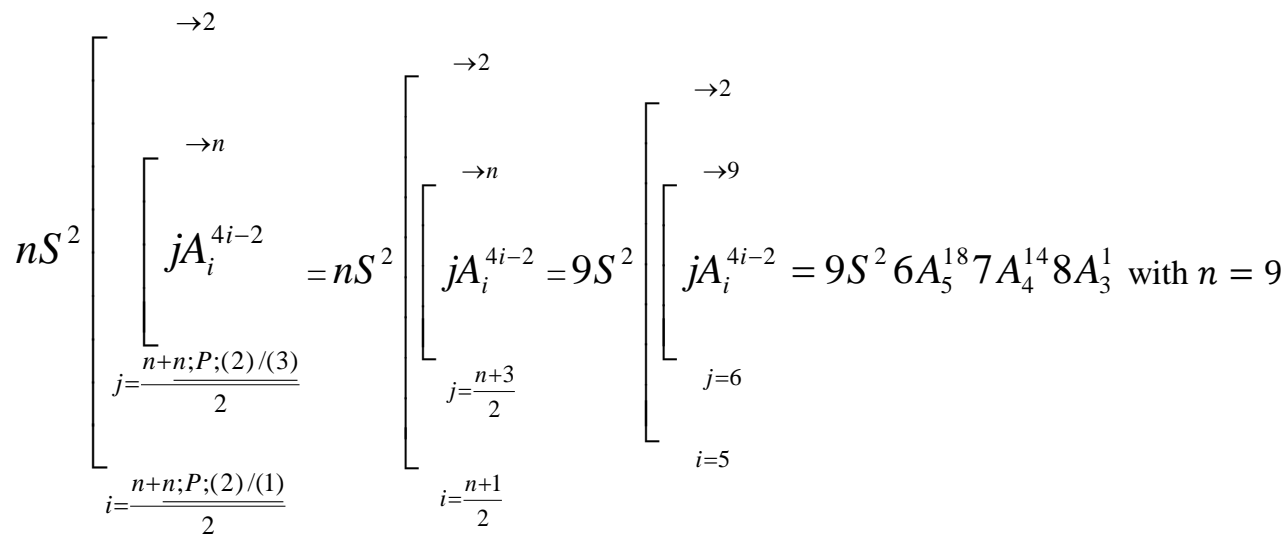

Briefly the Atom with $Z=203$ has the following characteristic:

$\circ$ Period $n=9$

- Number of peripheral electrons $N_{p e}=35$

- Group is $D^{9}=A_{3}^{1}$ (it's location is presented on the picture below)

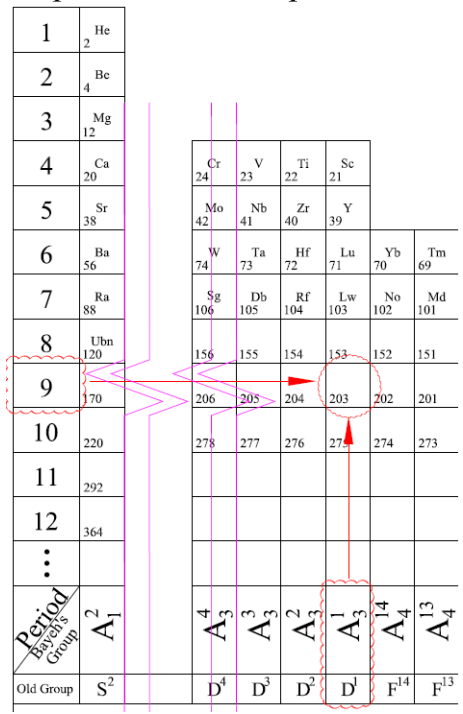

Figure 3: The Atom with $\mathrm{Z}=203$ is presented in the circle, it is in the period 8 and group $D^{1}$ or $A_{3}^{1}$ of the Bayeh's theoretical periodic table of elements.

\section{V.4. For $Z=218$}

Step 1:

By equation (6), we have to verify the equality

$\sum_{i=2}^{\sqrt{N}} i^{2}(P)==\frac{Z-2}{2}=\frac{218-2}{2}=108$

Step 2: 
We have to know exactly which case applies to the atom amongst one of the equations 13, 14, 15 or 16.

$\sum_{i=2}^{\sqrt{N}} i^{2}(P)==\left\{2 * 2^{2}+2 * 3^{2}+2 * 4^{2}+2 * 5^{2}=108=\frac{Z-2}{2}=108\right.$

In this case we can deduce that $P$ is equal to 2 therefore $v=P+1=3$ and $n=2 i-v ; P$; (2) $/(1) \Rightarrow n=2 *$ $5-1=9$ which is the period of the atom, therefore the period is odd.

Step 3:

Calculation of the number of peripheral electrons will be as the following;

$$
\begin{aligned}
N_{p e} & =Z-\left(2+2 \sum_{i=2}^{4} i^{2}(P)\right)=218-\left(2+2\left(\sum_{i=2}^{4} i^{2}(P)\right)\right. \\
& =218-\left(2+2\left(2 * 2^{2}+2 * 3^{2}+2 * 4^{2}+1 * 5^{2}\right)=50\right.
\end{aligned}
$$

Therefore the number of peripheral electrons is equal to $N_{p e}=50$.

Step 4:

The final step is to write the electron configuration of the last line (ground state electron configuration) As the number of peripheral electron is given, the same for the period, one can find easily the electron configuration.

$n S^{2}\left[\sum_{j=\frac{n+n ; P ;(2) /(3)}{2}}^{j A_{i}^{4 i-2}}=n S^{2}\left[\sum_{j=\frac{n+n ; P ;(2) /(1)}{2}}^{\rightarrow n}\left[A_{i}^{4 i-2}=9 S^{2}\right]\left[\begin{array}{l}\rightarrow 2 \\ j A_{i}^{4 i-2}=9 S^{2} 6 A_{5}^{18} 7 A_{4}^{14} 8 A_{3}^{10} 9 A_{2}^{6} \text { with } n=9 \\ j=\frac{n+1}{2}\end{array}\right.\right.\right.$

Briefly the Atom with $Z=218$ has the following characteristic

○ Period $n=9$

- Number of peripheral electrons $N_{p e}=50$

○ Group is $A_{2}^{6}$

\section{V.5. For $Z=365$}

Step 1:

By equation (6), we have to verify the equality

$\sum_{i=2}^{\sqrt{N}} i^{2}(P)==\frac{Z-2}{2}=\frac{365-2}{2}=181.5$

Step 2:

We have to know exactly which case applies to the atom amongst one of the equations $13,14,15$ or 16 .

$\sum_{i=2}^{\sqrt{N}} i^{2}(P)=\left\{\begin{array}{c}2 * 2^{2}+2 * 3^{2}+2 * 4^{2}+2 * 5^{2}+2 * 6^{2}=180<\frac{Z-2}{2}=181.5 \\ 2 * 2^{2}+2 * 3^{2}+2 * 4^{2}+2 * 5^{2}+2 * 6^{2}+1 * 7^{2}=229>\frac{Z-2}{2}=181.5\end{array}\right.$

In this case we can deduce that $P$ is equal to 1 therefore $v=P+1=2$ and $n=2 i-v ; P$; (2)/(1) $\Rightarrow n=2 *$ $7-2=12$ which is the period of the atom, therefore the period is even.

Step 3: 
Calculation of the number of peripheral electrons will be as the following;

$$
\begin{aligned}
N_{p e} & =Z-\left(2+2 \sum_{i=2}^{\sqrt{N}} i^{2}(P)\right)=365-\left(2+2\left(\sum_{i=2}^{6} i^{2}(P)\right)\right. \\
& =365-\left(2+2\left(2 * 2^{2}+2 * 3^{2}+2 * 4^{2}+2 * 5^{2}+2 * 6^{2}\right)=3\right.
\end{aligned}
$$

Therefore the number of peripheral electrons is equal to $N_{p e}=3$.

Step 4:

The final step is to write the electron configuration of the last line (ground state electron configuration). As the number of peripheral electron is given, the same for the period, one can find easily the electron configuration.

$$
n S^{2}\left[\sum_{j=\frac{n+n ; P ;(2) /(3)}{2}}^{j A_{i}^{4 i-2}}=n S^{2}\left[\sum_{j=\frac{n+n ; P ;(2) /(1)}{2}}^{\rightarrow n} j A_{i}^{4 i-2}=12 S^{2}\right]\left[\begin{array}{l}
\rightarrow 2 \\
j A_{i}^{4 i-2}=12 S^{2} 7 A_{7}^{1} \text { with } n=12 \\
j=\frac{n+2}{2}
\end{array}\right.\right.
$$

Briefly the Atom with $\mathrm{Z}=203$ has the following characteristic

$$
\begin{array}{ll}
\circ & \text { Period } n=12 \\
\circ & \text { Number of peripheral electrons } N_{p e}=3 \\
\circ & \text { Group is } A_{7}^{1}
\end{array}
$$

\section{V.6. For $Z=10452$}

Step 1:

By equation (6), we have to verify the equality

$\sum_{i=2}^{\sqrt{N}} i^{2}(P)==\frac{Z-2}{2}=\frac{10452-2}{2}=5225$

Step 2:

We have to know exactly which case applies to the atom amongst one of the equations $13,14,15$ or 16 .

$\sum_{i=2}^{\sqrt{N}} i^{2}(P)=\left\{\begin{array}{l}2 * 2^{2}+2 * 3^{2}+2 * 4^{2}+\cdots+2 * 19^{2}=4938<\frac{Z-2}{2}=5225 \\ 2 * 2^{2}+2 * 3^{2}+2 * 4^{2}+\cdots+2 * 19^{2}+1 * 20^{2}=5338>\frac{Z-2}{2}=5225\end{array}\right.$

In this case we can deduce that $P$ is equal to 1 therefore $v=P+1=2$ and $n=2 i-v ; P$; (2) /(1) $\Rightarrow n=2 *$ $20-2=38$ which is the period of the atom, therefore the period is even.

Step 3:

Calculation of the number of peripheral electrons will be as the following:

$$
\begin{aligned}
N_{p e} & =Z-\left(2+2 \sum_{i=2}^{\sqrt{N}} i^{2}(P)\right)=10452-\left(2+2\left(\sum_{i=2}^{19} i^{2}(P)\right)\right. \\
& =365-\left(2+2\left(2 * 2^{2}+2 * 3^{2}+2 * 4^{2}+2 * 5^{2}+\cdots+2 * 19^{2}\right)=574\right.
\end{aligned}
$$

Therefore the number of peripheral electrons is equal to $N_{p e}=574$.

Step 4: 
The final step is to write the electron configuration of the last line (ground state electron configuration). As the number of peripheral electron is given, the same for the period, one can find easily the electron configuration.

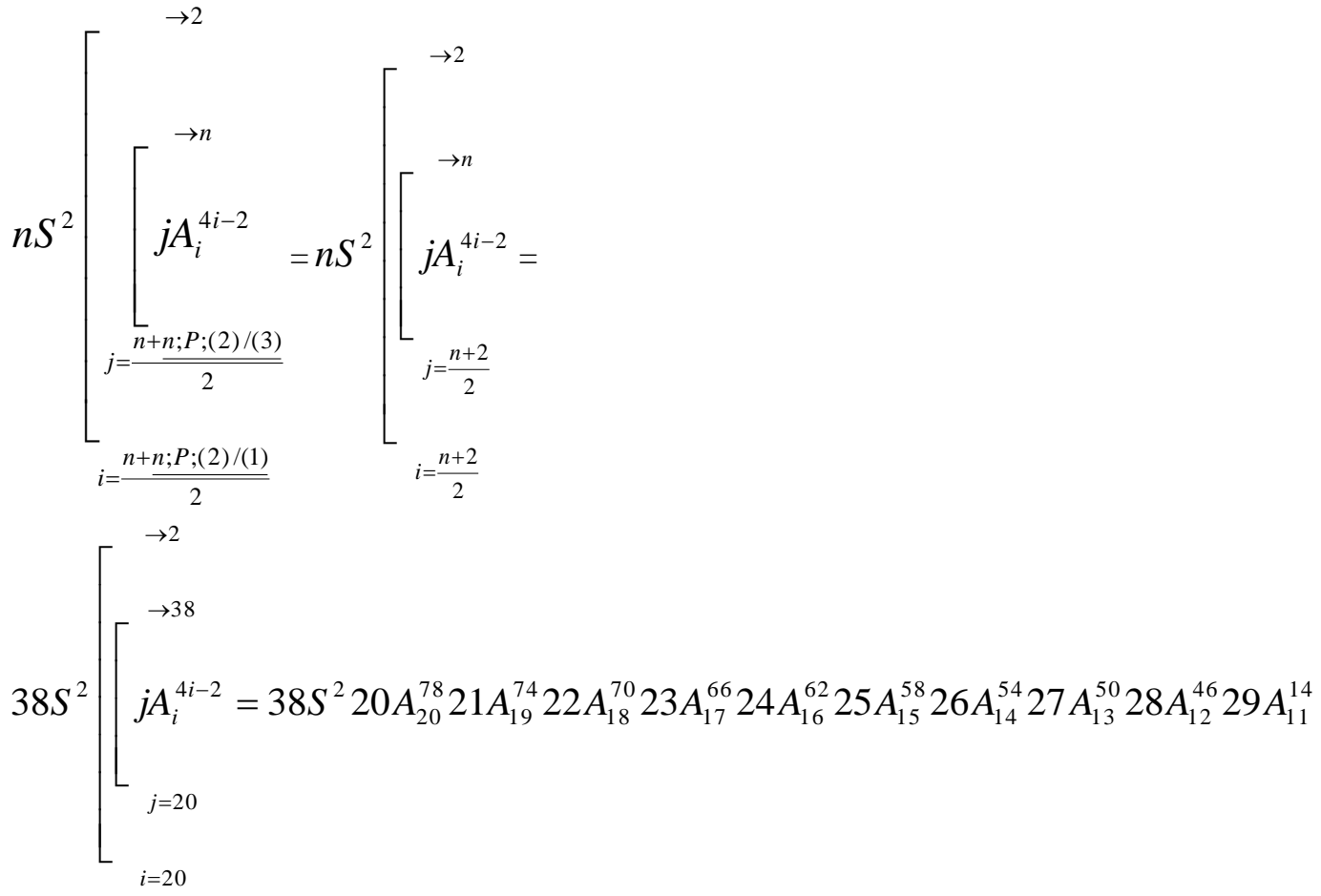

with $n=38$

Briefly the Atom with $Z=10452$ has the following characteristic

$\circ$ Period $n=38$

○ Number of peripheral electrons $N_{p e}=574$

○ Group is $A_{11}^{14}$

\section{Programming In C++ Language}

These formulae can be simply programmed. The following program is written in $\mathrm{C}++$ in order to facilitate the manipulation of the formulae introduced by the author and to visualize the results by a simple click. The user will obtain immediately results by just introducing the number $Z$ of the atom.

\#include $<$ cmath $>$

\#include<iostream>

using namespace std;

int $\mathrm{z}=0$; double $\mathrm{d}=0.0 ; \quad$ int $\mathrm{p}=1$; int $\mathrm{n}=2$; int $\mathrm{t}=0 ;$ int no; int $\mathrm{i}=0 ;$ int $\mathrm{j}=0 ;$ double somme $=0.0$; double som $=0.0$;

int main ()

$\{$ cout $<<"$ General Theory of electronic configuration of atoms, By Claude Ziad Bayeh in 2004/02/08"<<" Programmed by Claude Ziad Bayeh "<<endl; cout<<endl;

cout $<<"$ General theory of electron configuration" $<<$ endl $<<"$ of the ground state electron configuration" $<<$ endl $<<"$ The period and the number of electron for the peripheral line" $<<$ endl $<<"$ "<<endl;

cout $<<$ "Give the Atomic Number: "<<endl;

$\operatorname{cin}>>\mathrm{z}$;

while $(\mathrm{z}>=0)$

$\{\operatorname{switch}(\mathrm{z})$

$\{$ case 0 :cout $<<" 0 \mathrm{~s}(0) "<<$ endl; break;

case 1 :cout $<<" 1 \mathrm{~s}(1) "<<$ endl; break;

case 2 :cout $<<" 1 \mathrm{~s}(2) "<<$ endl;break;

default: $\mathrm{p}=1$;

$\mathrm{n}=2 ; \quad$ somme $=0.0 ; \quad$ som $=0.0 ; \quad \mathrm{d}=((($ static_cast $<$ double $>(\mathrm{z}))-2) / 2) ; \quad$ somme $+=\mathrm{p}^{*}$ pow $(\mathrm{n}, 2) ;$ for(int $\mathrm{k}=0 ; ;)\{\quad$ if $($ somme $<\mathrm{d})\{$ if $(\mathrm{p}==1)\{\mathrm{p}++$;som+=p*pow $(\mathrm{n}, 2)$;somme=som; $\}$ 


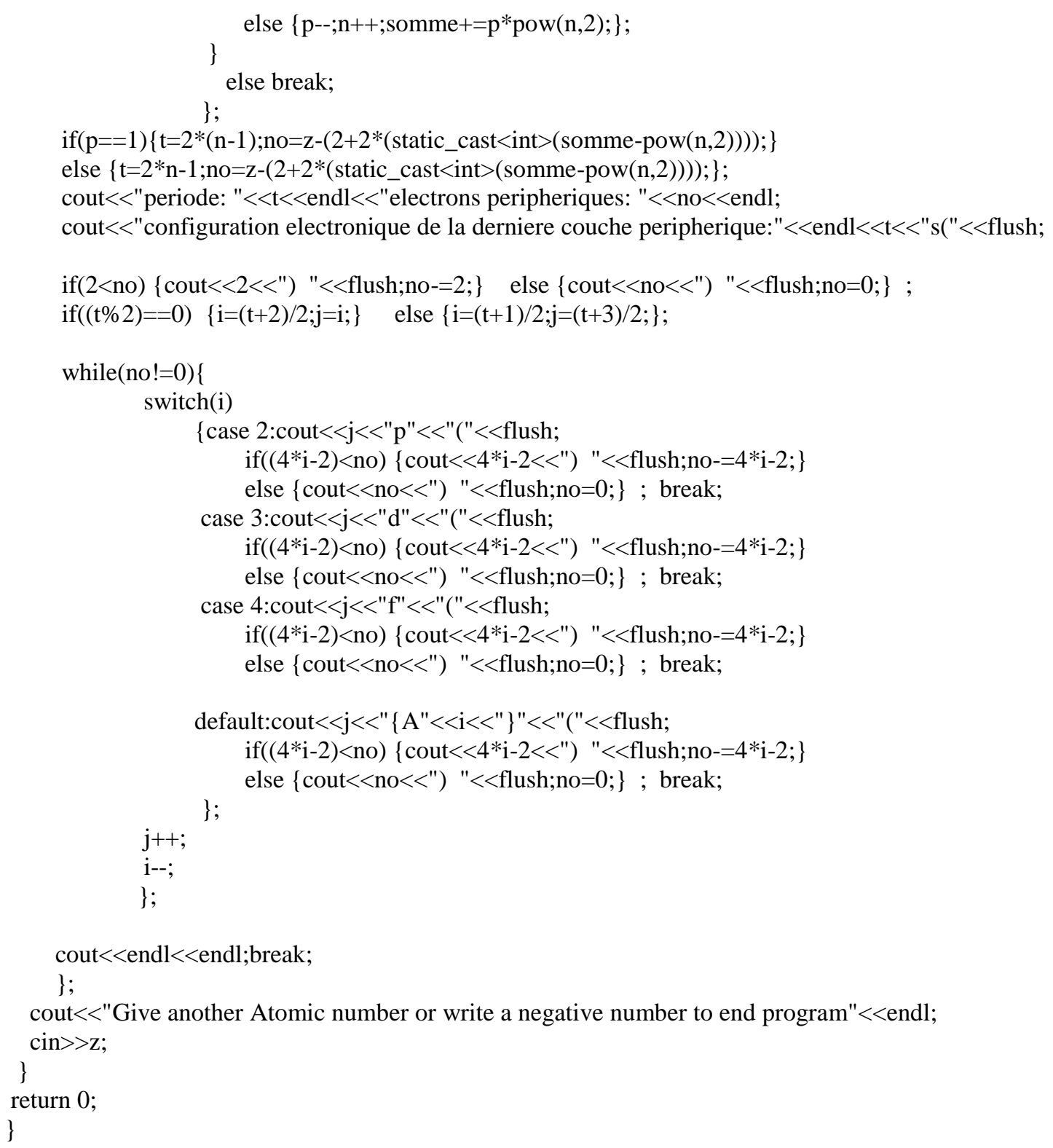

\section{PROGRAMMING IN Visual Basic.NET}

The formulae can be simply programmed using language Visual Basic.NET. The following program is written in VB.NET in order to simulate the result.

Note: The following script is a part of the complete program, because the other parts are programmed visually using special configurations which can't be written in total. In order to obtain the software, please contact the author.

'In the function we put :

Dim z As Integer $=0$

Dim d As Double $=0.0$

Dim $\mathrm{p}$ As Integer $=1$

Dim $n$ As Integer $=2$

Dim $t$ As Integer $=0$

Dim no As Integer

Dim i As Integer $=0$

Dim $\mathrm{j}$ As Integer $=0$

Dim somme As Double $=0.0$

Dim som As Double $=0.0$ 
Dim a As Integer $=1$

Dim output As String

$\mathrm{z}=$ TextBox1.Text

Select Case z

Case 0

MessageBox.Show("0s(0)", " Ground state electron configuration (Configuration electronique de la derniere couche)",MessageBoxButtons.OK)

Case 1

MessageBox.Show("1s(1)", " Ground state electron configuration (Configuration electronique de la derniere couche)",MessageBoxButtons.OK)

Case 2

MessageBox.Show("1s(2)", " Ground state electron configuration (Configuration electronique de la derniere couche)", MessageBoxButtons.OK)

Case Else

$\mathrm{p}=1$

$\mathrm{n}=2$

somme $=0.0$

som $=0.0$

$\mathrm{d}=((($ Convert.ToDouble $(\mathrm{z}))-2) / 2)$

somme $+=\mathrm{p} *$ Math.Pow $(\mathrm{n}, 2)$

While $\mathrm{a}=1$

If somme $<\mathrm{d}$ Then

If $\mathrm{p}=1$ Then

$\mathrm{p}=\mathrm{p}+1$

som $+=p *$ Math.Pow(n, 2)

somme $=$ som

Else

$\mathrm{p}=\mathrm{p}-1$

$\mathrm{n}=\mathrm{n}+1$

somme $+=p *$ Math.Pow $(n, 2)$

End If

Else

Exit While

End If

End While

If $\mathrm{p}=1$ Then

$\mathrm{t}=2 *(\mathrm{n}-1)$

no $=\mathrm{z}-(2+2 *($ Convert.ToInt32(somme - Math.Pow(n, 2)) ))

Else

$\mathrm{t}=2 * \mathrm{n}-1$

no $=\mathrm{z}-(2+2 *($ Convert.ToInt32(somme - Math.Pow $(\mathrm{n}, 2))))$

End If

output = "periode: " \& t \& vbCrLf \& "Peripheral electron (electrons peripheriques): " \& no \& vbCrLf output $\&=$ " Ground state electron configuration (configuration electronique de la derniere couche peripherique):" \& vbCrLf \& $\mathrm{t} \&$ "s("

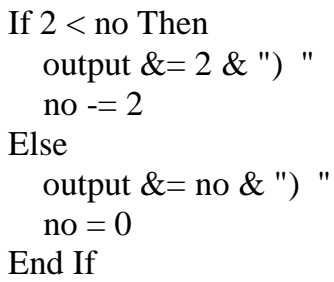




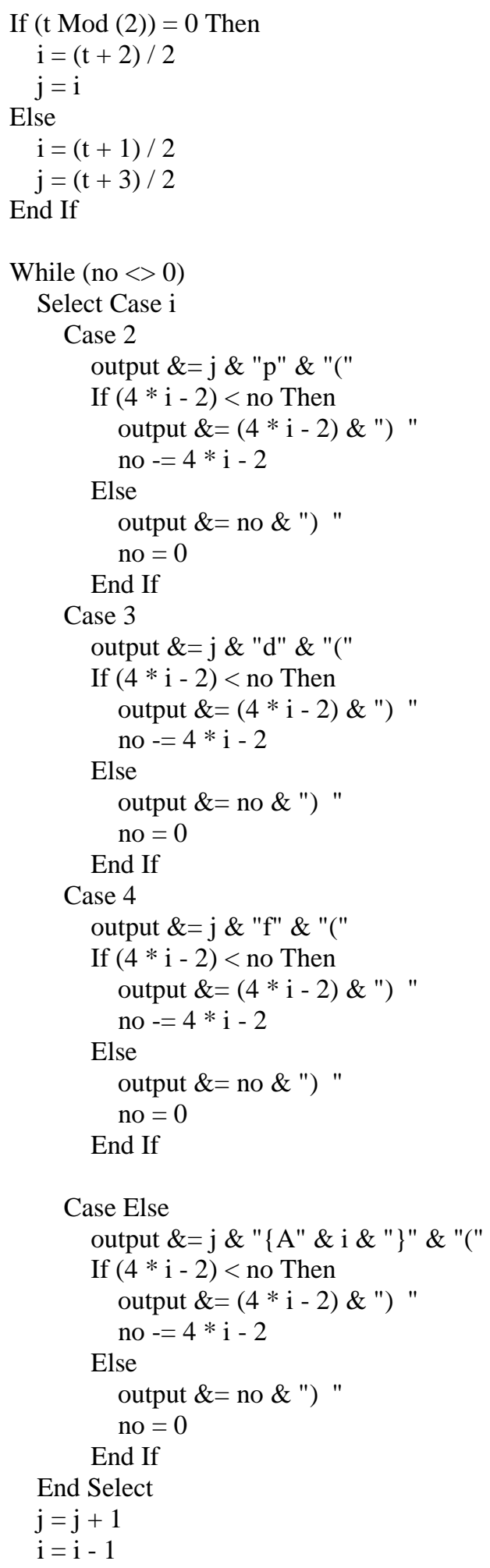

End While

output $\&=$ vbCrLf $\&$ vbCrLf

MessageBox.Show(output, " Ground state electron configuration (Configuration electronique de la derniere couche)", MessageBoxButtons.OK)

End Select 


\section{VII.1. Bayeh's Periodic table of Elements}

The following table is designed with VB.Net by the author and it can be considered the application of the theory on a periodic table. (This software is copyrighted, if you wish to order it, you must purchase it from the author directly).

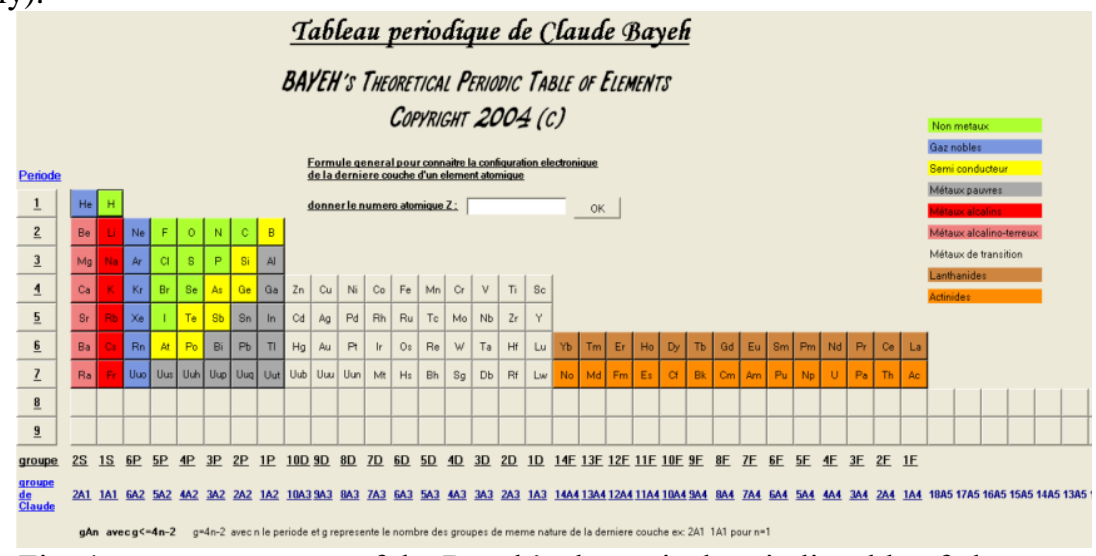

Fig. 4: represents a part of the Bayeh's theoretical periodic table of elements.

\section{VII.2. Results obtained using the software}

Note: The original software is written by the author in French language, so check the translation below the pictures for further understanding.

-If one pushes the button of an element, a window will appear and describe the element's characteristics, as the name of the atom, the Atomic number, the atomic mass....

For example if one pushes the Fluor box, the following window appears

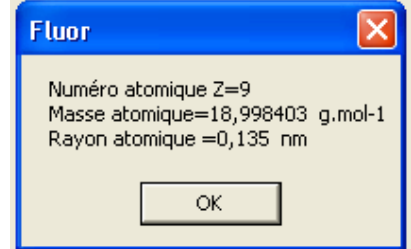

Fig. 5: The window that appears by pressing on the element

- There is a box that one can write the Atomic number and by clicking on the OK icon, results will appear as the following

\section{Formule qeneral pour connaitre la confiquration electronique}

de la derniere couche d'un element atomique

donner le numero atomique Z:

Fig. 5.1: Introducing the atomic number $\mathrm{Z}$ to the software.

Write the Atomic number 287 for example

\section{Formule qeneral pour connaitre la confiquration electronique}

de la derniere couche d'un element atomique

donner le numero atomique Z: 287

Fig. 5.2: Introducing the atomic number $\mathrm{Z}$ to the software.

The following results will appear

Configuration electronique de la derniere couche

periode: 10

electrons peripheriques: 69

configuration electronique de la derniere couche peripherique:

$10 s(2) 6\{A 6\}(22) 7\{A 5\}(18) 8 f(14) 9 d(10) 10 p(3)$

OK 
Fig. 6.1: represents the period, number of peripheral electrons, and electron configuration of an atom In this case, the period, the Peripheral number of electrons and the electronic configuration will appear.

- Example for $\mathrm{Z}=47$

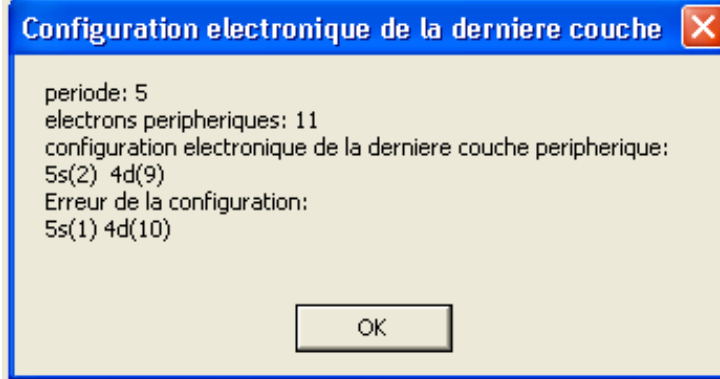

Fig. 6.2: represents the period, number of peripheral electrons, and electron configuration of an atom

- Example for $\mathrm{Z}=5420$

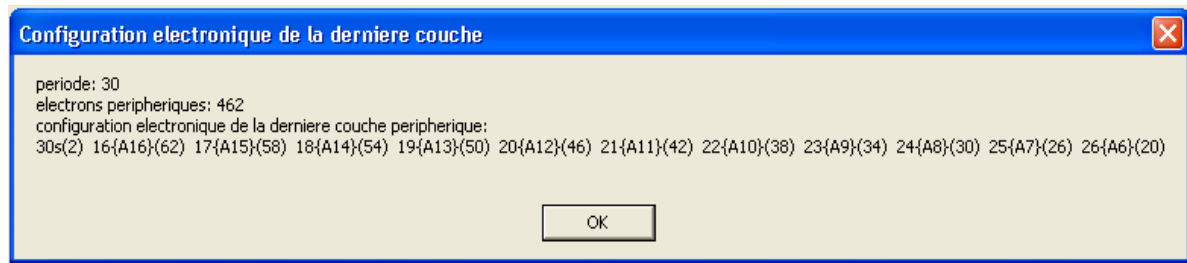

Fig. 6.3: represents the period, number of peripheral electrons, and electron configuration of an atom

- Example for $\mathrm{Z}=\mathrm{nm} 32$

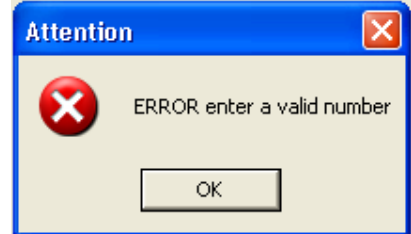

Fig. 7: Error window appears in case of a false data entry.

In this case as the data is not in integer form, so the program opens a window telling the error.

- The software is developed in a way that if one writes letters in the middle or at the end $(\mathrm{Z}=321 \mathrm{nmrfr} 2)$ the software will consider only the first digits as an input.

- If the entered number is decimal, then the program will consider the closest integer to the number:

E.g.: if $Z=34.4566$ is entered, the program takes $Z=34$,

If $Z=34.5566$ is entered, the program takes $Z=35$.

- One can know the group of the element from the ground state electron configuration (the last line electron configuration), it is based on the theoretical study and not on the practical study of the atom; for in the latter case, many errors are obtained naturally.

For example the atom with $\mathrm{Z}=47$ shown below, has two ground state configuration for the last line, the first configuration is obtained by theory as the study in this paper, while the second one represents the configuration error that is encountered by natural experience, in which is called error of the configuration. The theoretical configuration that appears is $\left(55 S^{2} 4 D^{9}\right)$, though in reality, this atom has errors in which the electron configuration is $\left(55 S^{1} 4 D^{10}\right)$. (refer to figure 6 )

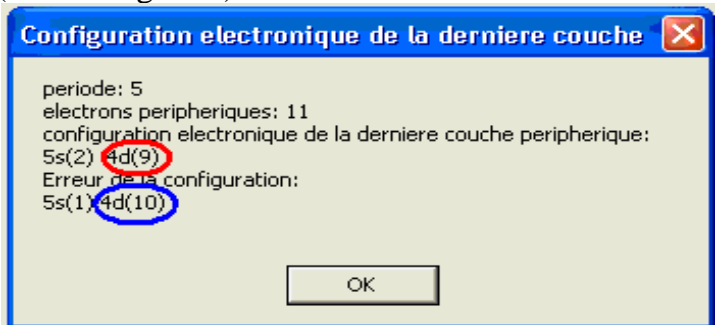

Fig. 8: Representing two electron configurations of the Atomic number 47: the theoretical configuration and the actual configuration. 


\section{Formulation Of The Theoretical Periodic Table}

The treated theory above can be applied by illustrating a theoretical periodic table that gives the theoretical positioning of the atom according the quantum physics, and then by applying the theory that is developed by the author. For a given Atomic number, the period and the electron configuration are known; therefore, one can know the exact location of the elements in the periodic table.

For example, by calculating the atomic number for $Z=47$, the obtained period is 5 and the configuration of the electron's last orbital is: $4 D^{9}$. Therefore, by locating the correct period (5), and the matching group $\left(4 D^{9}\right)$, the correct atom will be found on the following Theoretical Periodic Table.

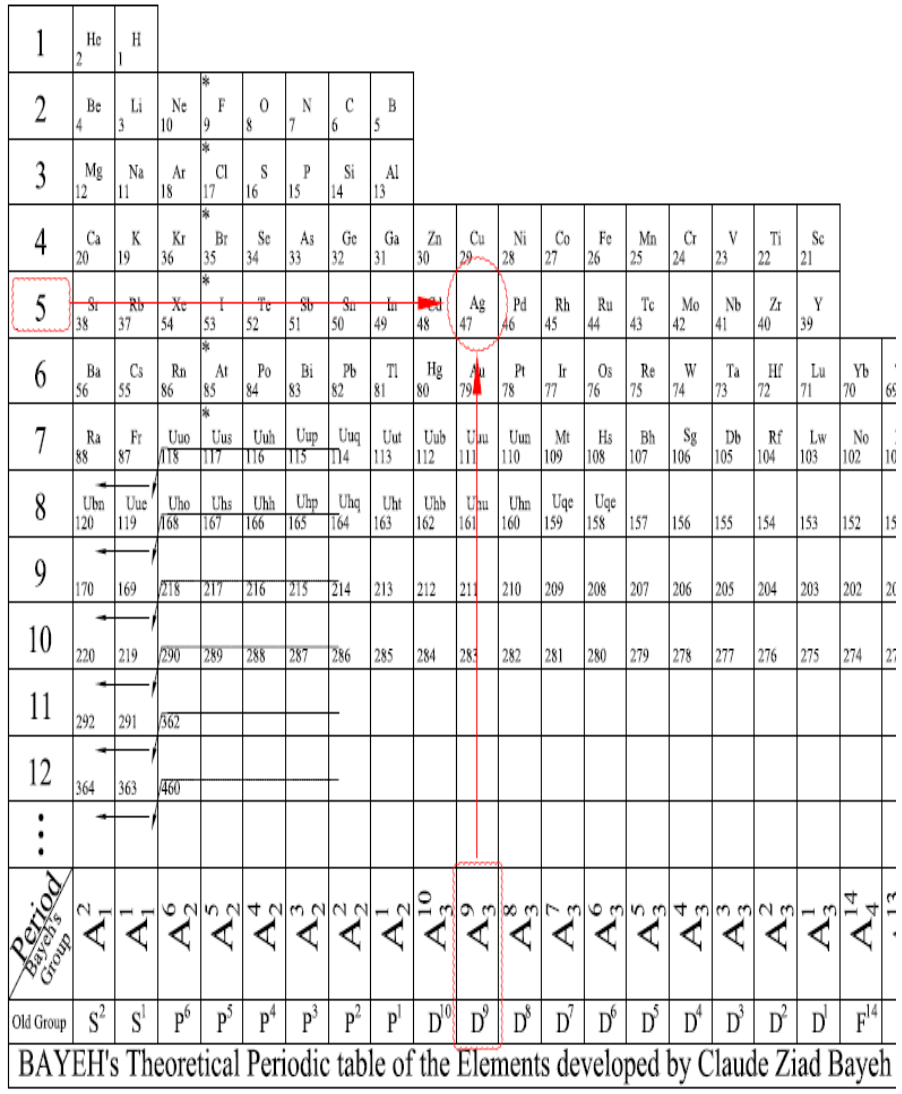

Fig. 9.1: Finding the position of an element, after having its period and last orbital's configuration.

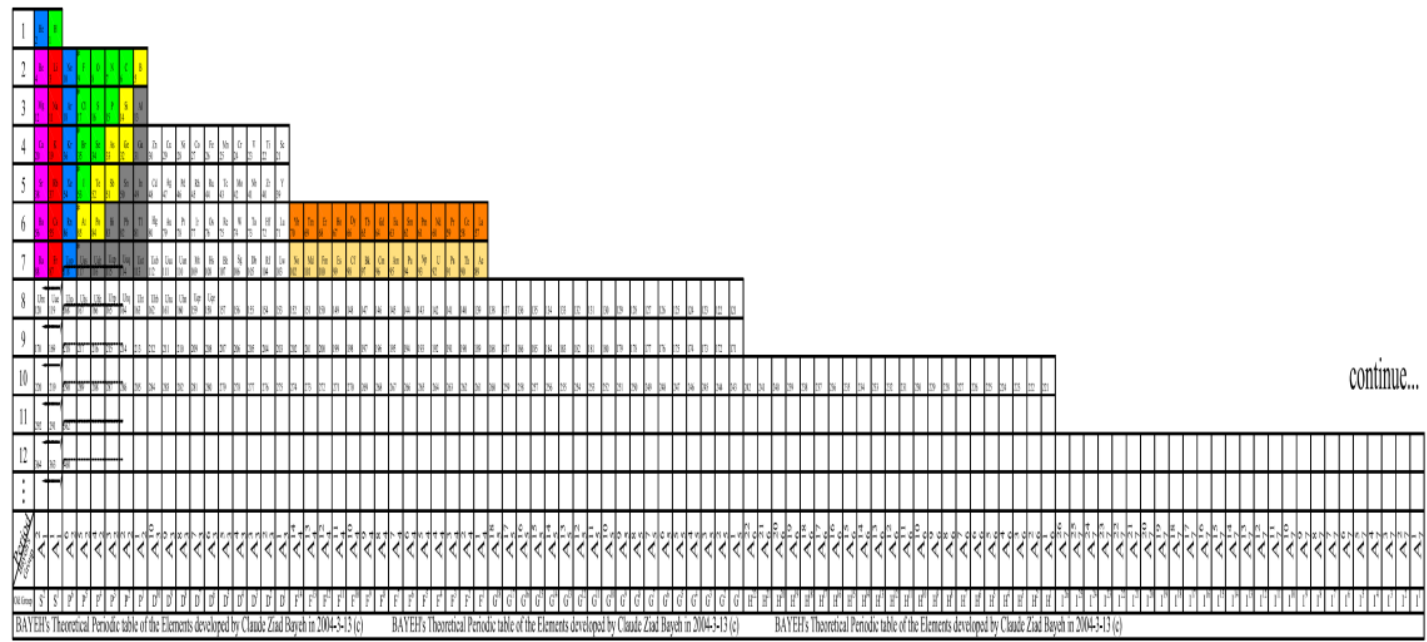

Fig. 9.2: Extracted part of the Bayeh's periodic table. 

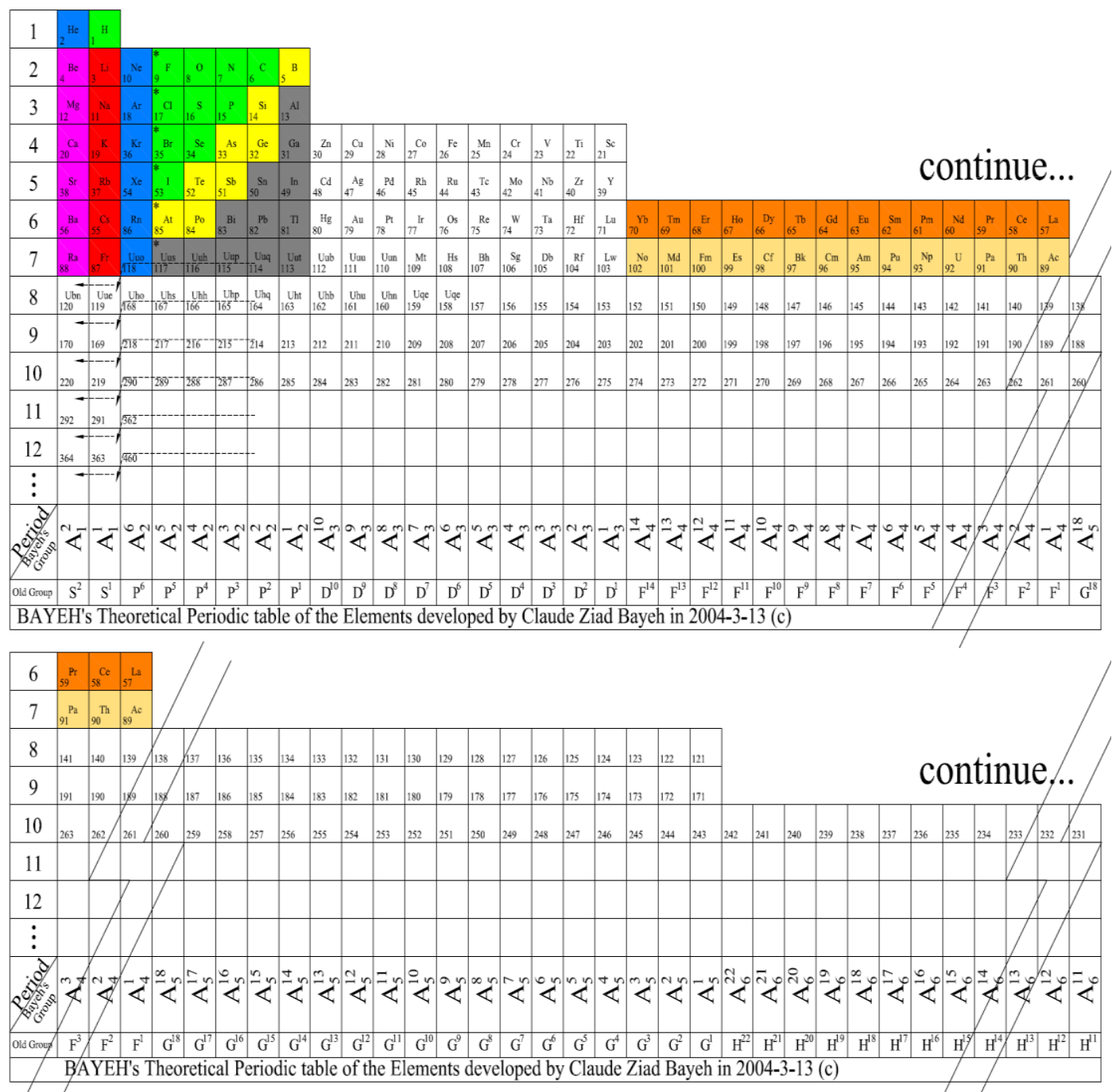

Fig. 9.3: A closer look at the first elements of Bayeh's Periodic Table.

LEGEND

\begin{tabular}{|c|c|c|c|c|}
\hline & JRE OF THE ELEMENT & Nomen & ature & \\
\hline * & & Numbc & Prefi & Letter \\
\hline & HALOGENS & 0 & nil & \\
\hline & & $\frac{1}{2}$ & un & $\mathrm{u}$ \\
\hline & TRANSITION METALS & $\frac{2}{3}$ & $\frac{b i}{t r i}$ & $\frac{b}{t}$ \\
\hline & METAUX DE TRANSITION & 4 & quad & $\frac{i}{q}$ \\
\hline & & 5 & pent & p \\
\hline & ACTINIDES & $\frac{6}{7}$ & hex & $\mathrm{h}$ \\
\hline & & $\frac{1}{8}$ & $\frac{\text { Sept }}{\text { oct }}$ & $\frac{\mathrm{s}}{\mathrm{o}}$ \\
\hline & LANTHANIDES & 9 & en & $\mathrm{e}$ \\
\hline & & NOTA & ION & \\
\hline & OTHER METALS & ${ }^{a S^{b}}=$ & & \\
\hline & METAUX PAUVRES & $\mathrm{aP}^{\mathrm{b}}=$ & & \\
\hline & $\begin{array}{l}\text { ALKALINE EAR IH MEIALS } \\
\text { METAUX ALCALINO-TERREUX }\end{array}$ & $\mathrm{aD}^{\mathrm{b}}=$ & & \\
\hline & ALKALINE METALS (ALKALI METALS) & $\mathrm{aF}^{\mathrm{b}}=$ & & \\
\hline & METAUX ALCALINS & $\mathrm{aG}^{\mathrm{b}}=$ & & \\
\hline & NOBLE GASES & $\mathrm{aH}^{\mathrm{b}}=$ & & \\
\hline & UAZ NUBLES & 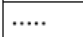 & & \\
\hline & NON METAL (NON METAUX) & & & \\
\hline & $\begin{array}{l}\text { METALLOIDS } \\
\text { SEMI CONDUCTEUR }\end{array}$ & & $\begin{array}{l}\text { ture of } \\
\text { c numbe }\end{array}$ & $\begin{array}{l}\text { the } \\
\text { e element }\end{array}$ \\
\hline
\end{tabular}

Fig. 9.4: Legend of the periodic table 


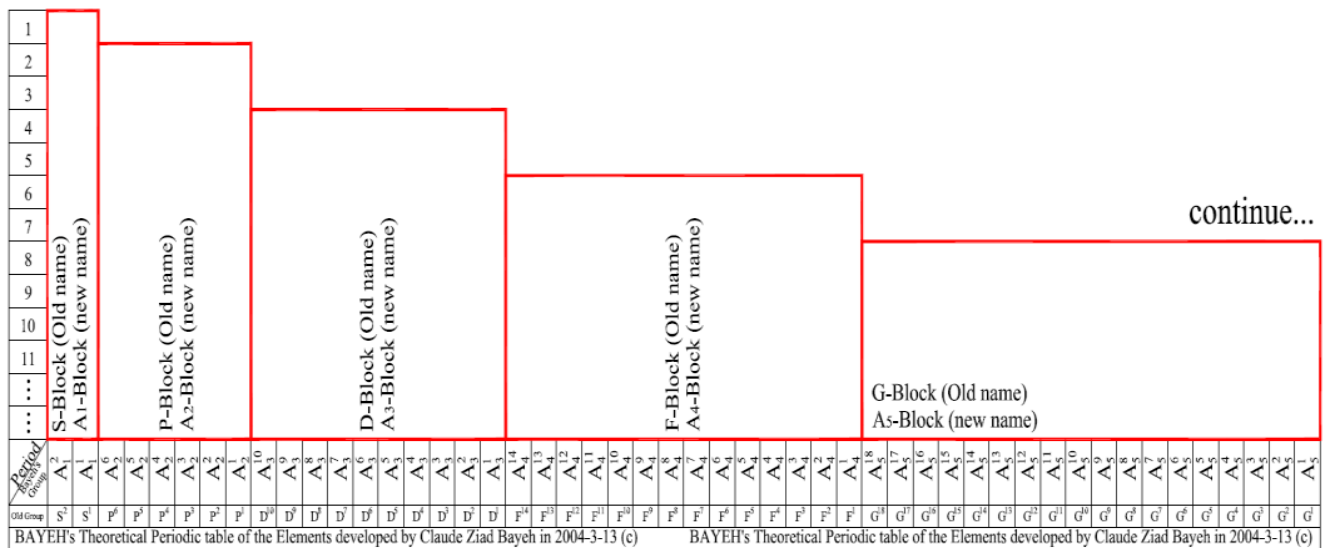

Fig. 9.5: Theoretical periodic table arranged by sequential Blocks with respective Groups number.

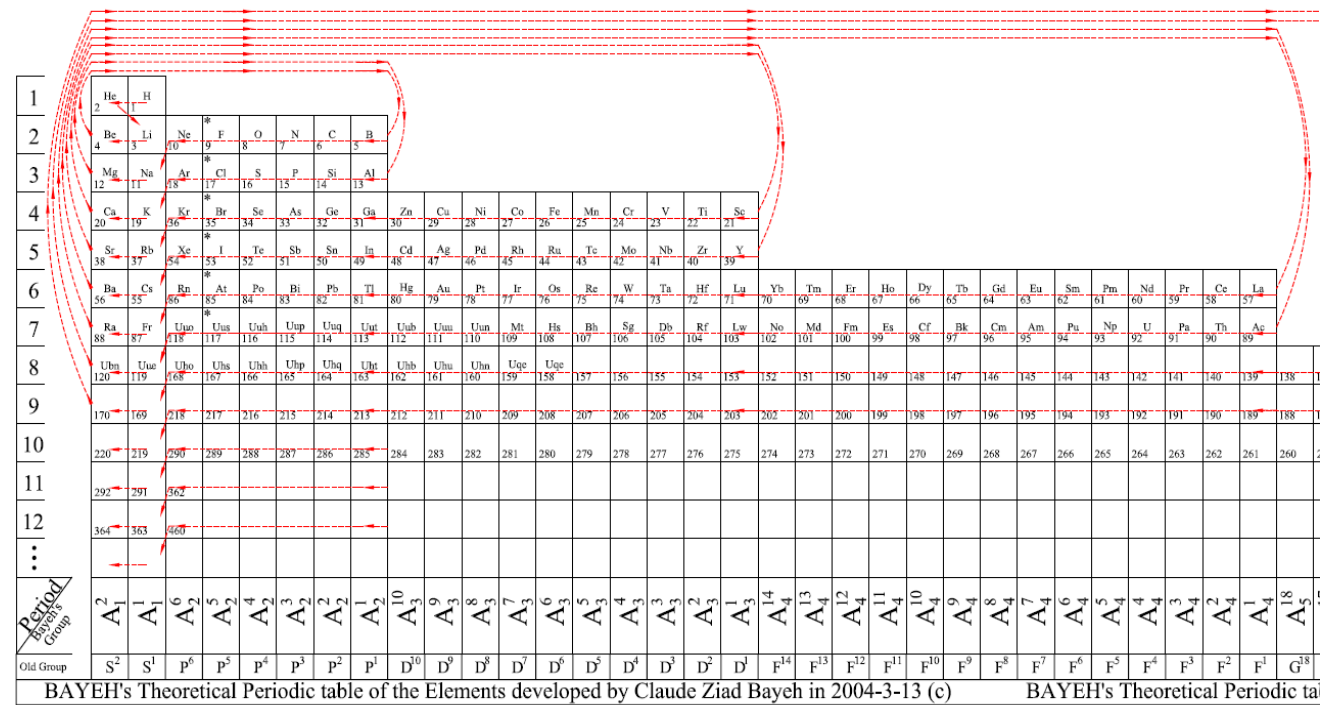

Fig. 9.6: Theoretical periodic table arranged by sequential atomic numbers.

\section{Conclusion}

As we have seen in this paper, the importance of this theory is in facilitating the process of calculation. In fact, this theory can be used to obtain information about the atom (like its ground state electron's configuration, its period, group, number of electron configuration, etc...). And this method can be applied to give precise answers, with a minimum calculation time.

The author has also developed this theory in two different programming languages as $\mathrm{C}++$ and VB.NET, in order to allow the student to copy and simulate it on his own and obtain accurate results.

In addition, and based on the same theory, the author has also developed a new periodic table which it is explained in the articles named "Bayeh's theoretical periodic table" [3]. (It is also presented in chapter 2 in this book).

\section{References}

[1] D.H. Andrews and R.J. Kokes, Notions fondamentales de chimie, DUNOD Paris, (1968), pp. 1-120

[2] A. Dingle and S. Basher, The Periodic Table: Elements with Style, Kingfisher, ISBN 0753460858, (2007), pp.1-128.

[3] Bohr Niels, The Quantum Postulate and the Recent Development of Atomic Theory, Nature 121 (3050): Bibcode 1928 Natur.121..580B. doi:10.1038/121580a0, (April 1928), pp.580-590.

[4] Richard E. Powell, The five equivalent d orbitals, Journal of Chemical Education 45 (1): (1968), pp.45.

[5] W.C. Martin and W.L. Wiese, Atomic Spectroscopy, in: Atomic, Molecular, \& Optical Physics Handbook, Eds. G.W.F. Drake (AIP, Woodbury, NY, 1996) Chapter 10, pp. 135-153.

[6] M. E. Wieser, Atomic weights of the elements 2005 (IUPAC Technical Report), Pure Appl. Chem. (IUPAC) 78 (11), (2006), pp. 2051-2066.

[7] M. E. Wieser, IUPAC Standard Atomic Weights Revised (2007). IUPAC. (2007).

[8] Francis Halzen and Alan D. Martin, QUARKS AND LEPTONS: An Introductory Course in Modern Particle Physics, John Wiley \& Sons. ISBN 0-471-88741-2, (1984).

[9] Paul A.M. Dirac, Principles of quantum mechanics, Oxford University Press, ISBN 0-19-852011-5, (1982). 
[10] Eyring Henry, Quantum Chemistry, the late John Walter and George E.Kimball John Wiley and Sons, ISBN:0-471-24981-5, (2006), pp. 1-50

[11] Kunming Xu, The unification of Pythagorean Theorem for electronic orbitals with Kepler's law for planetary orbits, Scientific Research Monthly, (2006), pp.1-17.

[12] Primo Levi, The Periodic Table, Schocken; Translated By Raymond Rosenthal, ISBN-10: 0805210415, ISBN-13: 978-0805210415, (April 4, 1995), pp. 1-240.

[13] M. R. Kibler, From the Mendeleev periodic table to particle physics and back to the periodic table, Foundations of Chemistry 9 , (2007), pp. 221-234, arXiv:quant-ph/0611287v2.

[14] M. R. Kibler, A Group-Theoretical Approach to the Periodic Table of Chemical Elements: Old and New Developments, in: "The Mathematics of Periodic Table”, D.H. Rouvray and R.B. King, Eds. (Nova Science Publishers, New York, 2006), pp. 237-263, arXiv:quant-ph/0503039v1.

[15] M. R. Kibler, On the use of the group SO(4,2) in atomic and molecular physics Molecular Physics 102, (2004), pp.1221-1229, arXiv:quant-ph/0409209v1.

[16] M. R. Kibler, On a group-theoretical approach to the periodic table of chemical elements, in: "Symmetry Methods in Physics XI", C. Burdik, O. Navratil and S. Posta, Eds. (Joint institute for Nuclear, Dubna, 2004), arXiv:quant-ph/0408104v1.

[17] M. R. Kibler, Classifying chemical elements and particles: from the atomic to the sub-atomic world, in: "The Periodic Table: Into the 21st Century", D.H. Rouvray and R.B. King, Eds. (Research Studies Press, Baldock, UK, 2004), pp. 297-329, arXiv:quantph/ $0310155 \mathrm{v} 2$

[18] T. Nigadi and M. R. Kibler The Periodic Table in Flatland, Int. J. Quantum Chem. 57 (1996), pp. 53-61, arXiv:atom-ph/9512001v1.

[19] George B. Arfken and Hans J. Weber, Mathematical Methods for Physicists, Elsevier Academic Press, ISBN 0120598760, (2005), p. 743 .

[20] S. L. Belousov, Tables of normalized associated Legendre polynomials, Mathematical tables, Pergamon Press, (1962), pp.18.

[21] T. M. Dunster, , Legendre and Related Functions, in Olver, Frank W. J.; Lozier, Daniel M.; Boisvert, Ronald F. et al., NIST Handbook of Mathematical Functions, Cambridge University Press, ISBN 978-0521192255, (2010).

[22] Milton Orchin, Roger S. Macomber, Allan Pinhas, and R. Marshall Wilson, Atomic Orbital Theory, (2005).

[23] J. Daintith, Oxford Dictionary of Chemistry, New York: Oxford University Press, ISBN 0-19-860918-3, (2004).

[24] David Griffiths, Introduction to Quantum Mechanics, Prentice Hall, ISBN 0-13-124405-1, (1995), pp. 190-191.

[25] Niels Bohr, On the Constitution of Atoms and Molecules, Philosophical Magazine 26 (1): (1913), pp.476. http://www.chemteam.info/Chem-History/Bohr/Bohr-1913a.html

[26] Claude Ziad Bayeh, "Introduction to the Infomath function in mathematics and its application in electrical and electronic engineering", WSEAS Transactions on Advances in Engineering Education, E-ISSN: 2224-3410, Issue 1, Volume 10, January 2013, pp. 24-36. 